\title{
GENERAL DE FINETTI TYPE THEOREMS IN NONCOMMUTATIVE PROBABILITY
}

\author{
WEIHUA LIU
}

\begin{abstract}
We prove general de Finetti type theorems for classical and free independence. The de Finetti type theorems work for all non-easy quantum groups, which generalize a recent work of Banica, Curran and Speicher. We determine maximal distributional symmetries which means the corresponding de Finetti type theorem fails if a sequence of random variables satisfy more symmetry relations other than the maximal one. In addition, we define Boolean quantum semigroups in analogous to the easy quantum groups, by universal conditions on matrix coordinate generators and an orthogonal projection. Then, we show a general de Finetti type theorem for Boolean independence.
\end{abstract}

\section{INTRODUCTION}

The area of distributional symmetries is one of the richest of modern probability theory. The most obvious problem in this area is to characterize the class of objects of a given type with a specified symmetric property of their joint distribution. For example, de Finetti's fundamental theorem states that an infinite sequence of random variables, whose joint distribution is invariant under all finite permutations, is conditionally independent and identically distributed. Later, in [6], rotatability and other continuous symmetries were considered by Freedman. One can see [8] for more details.

Exchangeability and rotatability are classical symmetries associated with permutation groups and orthogonal groups. The quantum analogue of permutation and orthogonal groups were introduced by Wang [23, 24]. They are compact quantum groups in the sense of Woronowicz's matrix pseudogroups [26, 27]. By using symmetries associated with quantum permutation groups, Köstler and Speicher discovered a free analogue of classical de Finetti theorem [10]: an infinite sequence of noncommutative random variables are invariant under quantum permutations is equivalent to the fact that the random variables are identically distributed and free with respect to the conditional expectation onto their tail algebra. A free analogue of Freedman's work on rotatability was given by Curran in [5].

In [4], both classical symmetries and quantum symmetries are studied in the "easiness" formalism. Roughly speaking, those structures are quantum groups associated with tensor categories of partitions. For each $n$, it was shown that there are exactly six easy groups which are denoted by $S_{n}$, $O_{n}, B_{n}, H_{n}, B_{n}^{\prime}$ and $S_{n}^{\prime}$. We will denote the algebras of continuous functions on these groups by $C_{s}(n), C_{o}(n), C_{b}(n), C_{h}(n), C_{b^{\prime}}(n)$ and $C_{s^{\prime}}(n)$, respectively. In the quantum aspects, for each $n$, together with a work of Weber 25], there are exactly seven easy quantum groups which are denoted by $A_{s}(n), A_{o}(n), A_{b}(n), A_{h}(n), A_{s^{\prime}}(n), A_{b^{\prime}}(n)$ and $A_{b^{\#}}(n)$. All these algebras are generated by $n^{2}$ matrix coordinates $\left\{u_{i, j} \mid i, j=1, \ldots, n\right\}$ which satisfy certain relation $R$. The relations $R$ for $C_{*}(n)$ and $A_{*}(n)$ are suitable such that all these algebras are Hopf $C^{*}$-algebras in the sense of Woronowicz [26]. The distributional symmetries associated with Woronowicz's $C^{*}$-algebras are defined via coactions of quantum groups on noncommutative polynomials in the sense of Soltan [16]. Among these symmetries, Banica, Curran and Speicher studied de Finetti type theorems for $C_{s}(n), C_{o}(n), C_{b}(n), C_{h}(n)$ and $A_{s}(n), A_{o}(n), A_{b}(n), A_{h}(n)$ [3]. In 
short, these symmetries can characterize independence relations which are classical or free. Furthermore, it determines some special distributions which are symmetric, shifted central limit or centered central limit laws. One goal of this paper is to study de Finetti type theorems for all compact quantum groups which are either between $C_{s}(n)$ and $C_{o}(n)$ or between $A_{s}(n)$ and $A_{o}(n)$.

In [15], Ryll-Nardzewski showed that de Finetti theorem holds under the weaker condition of spreadability. It shows that different kinds of distributional symmetries may give the same de Finetti type theorem. In this paper, we study when different kinds of distributional symmetries give the same de Finetti type theorem. We show that there is no distribution other than what $C_{s}(n), C_{o}(n), C_{b}(n), C_{h}(n)$ and $A_{s}(n), A_{o}(n), A_{b}(n), A_{h}(n)$ can characterize. On the other hand, we will show that these distributional symmetries are maximal which means the corresponding de Finetti type theorem fails if a sequence of random variables satisfy more symmetries other than the maximal one.

In 18, 20, it was shown that there is a unique non-unital independence, which is called Boolean independence, in noncommutative probability. The study of distributional symmetries for Boolean independence was started in [12]. We constructed a family of quantum semigroups in analogues to Wang's quantum permutation groups and defined their coactions on the joint distribution of a sequence of random variables. It was shown that the distributional symmetries associated with those coactions can be used to characterize Boolean independence in a proper framework. In a recent work of Hayase [7], following the idea of Banica and Speicher, many distributional symmetries related to Boolean independence were constructed via the category of interval partitions. By using those distributional symmetries, Hayase found de Finetti type theorems for a Boolean analogue of the easy quantum groups. We will define quantum semigroups, which are related to Boolean independence, in analogous to the easy quantum groups via some universal conditions. The general de Finetti type theorem for Boolean independence is not complete. However, with the method introduced in the paper, it is still possible to check de Finetti type theorems for some quantum semigroups other than the Boolean analogue of the easy quantum groups.

In the following general de Finetti type theorems, $A \subseteq B$ can be understood that all distributional symmetries associated with $A$ are distributional symmetries associated with $B$. Unlike the unified theorem in [3, we divide our de Finetti type theorems in to two cases since we should take account of Gaussian distributions whose support is non-compact.

Theorem 1.1. Let $(\Omega, \Sigma, \mu)$ be a classical probability space and $\left(x_{i}\right)_{i \in \mathbb{N}}$ be a sequence of realvalued random variables such that $x_{i} \in \bigcap_{1 \leq p<\infty} L^{p}(\Omega, d \mu)$ for all $i$. Let $\mathcal{A}$ be the algebra of all complex-valued random variables and $\phi$ is the classical expectation. Assume that $\left(x_{i}\right)_{i \in \mathbb{N}}$ generate $\mathcal{A}$. Let $\{E(n)\}_{n \in \mathbb{N}}$ be a sequence of orthogonal Hopf algebras such that $C_{s}(n) \subseteq$ $E(n) \subseteq C_{o}(n)$ for each $n \in \mathbb{N}$. If the joint distribution of $\left(x_{i}\right)_{i \in \mathbb{N}}$ is $E(n)$-invariant, then there are a subalgebra $1 \subseteq \mathcal{B} \subseteq \mathcal{A}$ and a $\phi$-preserving conditional expectation $\mathbb{E}: \mathcal{A} \rightarrow \mathcal{B}$ such that

1. If $E(n)=C_{s}(n)$ for all $n$, then $\left(x_{i}\right)_{i \in \mathbb{N}}$ are conditionally independent and identically distributed with respect to $\mathbb{E}$.

2. If $C_{s}(n) \subseteq E(n) \subseteq C_{h}(n)$ for all $n$ and there exists a $k$ such that $E(k) \neq C_{s}(k)$, then $\left(x_{i}\right)_{i \in \mathbb{N}}$ are conditionally independent and have identical symmetric distribution with respect to $\mathbb{E}$.

3. If $C_{s}(n) \subseteq E(n) \subseteq C_{b}(n)$ for all $n$ and there exists a $k$ such that $E(k) \neq C_{s}(k)$, then $\left(x_{i}\right)_{i \in \mathbb{N}}$ are conditionally independent and have identical shifted-Gaussian distribution with respect to $\mathbb{E}$. 
4. If there exist $k_{1}, k_{2}$ such that $E\left(k_{1}\right) \nsubseteq C_{h}\left(k_{1}\right)$ and $E\left(k_{2}\right) \nsubseteq C_{b}\left(k_{2}\right)$, then $\left(x_{i}\right)_{i \in \mathbb{N}}$ are conditionally independent and have centered Gaussian distribution with respect to $\mathbb{E}$.

The following theorem is for noncommutative probability. We will only consider bounded random variables.

Theorem 1.2. Let $(\mathcal{A}, \phi)$ be a $W^{*}$-probability space and $\left(x_{i}\right)_{i \in \mathbb{N}}$ be a sequence of random variables which generate $\mathcal{A}$.

- Suppose that $\phi$ is faithful. Let $\{E(n)\}_{n \in \mathbb{N}}$ be a sequence of orthogonal Hopf algebras such that $A_{s}(n) \subseteq E(n) \subseteq A_{o}(n)$ for each $n$. If the joint distribution of $\left(x_{i}\right)_{i \in \mathbb{N}}$ is $E(n)$ invariant, then there are a $W^{*}$-subalgebra $1 \subseteq \mathcal{B} \subseteq \mathcal{A}$ and a $\phi$-preserving conditional expectation $\mathbb{E}: \mathcal{A} \rightarrow \mathcal{B}$ such that

1. If $E(n)=A_{s}(n)$ for all $n$, then $\left(x_{i}\right)_{i \in \mathbb{N}}$ are freely independent and identically distributed with respect to $\mathbb{E}$.

2. If $A_{s}(n) \subseteq E(n) \subseteq A_{h}(n)$ for all $n$ and there exists a $k$ such that $E(k) \neq A_{s}(k)$, then $\left(x_{i}\right)_{i \in \mathbb{N}}$ are freely independent and have identical symmetric distribution with respect to $\mathbb{E}$.

3. If $A_{s}(n) \subseteq E(n) \subseteq A_{b}(n)$ for all $n$ and there exists a $k$ such that $E(k) \neq A_{s}(k)$, then $\left(x_{i}\right)_{i \in \mathbb{N}}$ are conditionally independent and have identical shifted-semicircular distribution with respect to $\mathbb{E}$.

4. If there exist $k_{1}, k_{2}$ such that $E\left(k_{1}\right) \nsubseteq A_{h}\left(k_{1}\right)$ and $E\left(k_{2}\right) \nsubseteq A_{b}\left(k_{2}\right)$, then $\left(x_{i}\right)_{i \in \mathbb{N}}$ are freely independent and have centered semicircular distribution with respect to $\mathbb{E}$.

- Suppose that $\phi$ is non-degenerated. Let $\{E(n)\}_{n \in \mathbb{N}}$ be a sequence of orthogonal Boolean quantum semigroups such that $B_{s}(n) \subseteq E(n) \subseteq B_{o}(n)$ for each $n$. If the joint distribution of $\left(x_{i}\right)_{i \in \mathbb{N}}$ is $E(n)$-invariant, then there are a $W^{*}$-subalgebra (does not necessarily contains the unit of $\mathcal{A}) \mathcal{B} \subseteq \mathcal{A}$ and a $\phi$-preserving conditional expectation $\mathbb{E}: \mathcal{A} \rightarrow \mathcal{B}$ such that

1. If $E(n)=B_{s}(n)$ for all $n$, then $\left(x_{i}\right)_{i \in \mathbb{N}}$ are Boolean independent and identically distributed with respect to $\mathbb{E}$.

2. If $B_{s}(n) \subseteq E(n) \subseteq B_{h}(n)$ for all $n$ and there exists a $k$ such that $E(k)$ has a quotient algebra $E^{\prime}(k)$ that $A_{s}(k) \nsubseteq E^{\prime}(k) \subseteq A_{n}(n)$, then $\left(x_{i}\right)_{i \in \mathbb{N}}$ are Boolean independent and have identical symmetric distribution with respect to $\mathbb{E}$.

3. If $B_{s}(n) \subseteq E(n) \subseteq B_{b}(n)$ for all $n$ and there exists a $k$ such that $E(k)$ has a quotient algebra $E^{\prime}(k)$ that $A_{s}(k) \nsubseteq E^{\prime}(k) \subseteq A_{b}(n)$, then $\left(x_{i}\right)_{i \in \mathbb{N}}$ are Boolean independent and have identical shifted-Bernoulli distribution with respect to $\mathbb{E}$.

4. If there exist $k_{1}, k_{2}$ such that $E\left(k_{1}\right)$ and $E\left(k_{2}\right)$ have quotient algebras $E^{\prime}\left(k_{1}\right) \subseteq$ $A_{o}\left(k_{1}\right)$ and $E^{\prime}\left(k_{2}\right) \subseteq A_{o}\left(k_{2}\right)$ such that $E\left(k_{1}\right) \nsubseteq A_{h}\left(k_{1}\right)$ and $E^{\prime}\left(k_{2}\right) \nsubseteq A_{b}\left(k_{2}\right)$, then $\left(x_{i}\right)_{i \in \mathbb{N}}$ are conditionally independent and have centered Bernoulli distribution with respect to $\mathbb{E}$.

Besides this introductory section, the rest of this paper is organized as follows. In Section 2 , we recall some definitions in noncommutative probability and some combinatorial tools. In Section 3, we recall the orthogonal Hopf algebras and study their properties. In Section 4, we define Boolean quantum semigroups in analogous to the easy quantum groups via certain universal conditions. In Section 5, we give the proof of our main theorems and some applications.

\section{Preliminaries AND examples}

In this section, we recall some necessary definitions and notation in noncommutative probability. For further details, see texts [10, 12, 13, 22]. 
2.1. Noncommutative probability. This part is for noncommutative probability theory and universal independence relations.

Definition 2.1. A noncommutative probability space is a pair $(\mathcal{A}, \phi)$, where $\mathcal{A}$ is a unital algebra and $\phi: \mathcal{A} \rightarrow \mathbb{C}$ is a linear functional such that $\phi\left(1_{\mathcal{A}}\right)=1 .(\mathcal{A}, \phi)$ is a commutative probability space if $\mathcal{A}$ is commutative. $(\mathcal{A}, \phi)$ is a $C^{*}$-probability space if $\mathcal{A}$ is a $C^{*}$-algebra and $\phi$ is a state, i.e. norm one positive linear functional. $(\mathcal{A}, \phi)$ is a $W^{*}$-probability space if $\mathcal{A}$ is a $W^{*}$-algebra and $\phi$ is a normal state. $\phi$ is tracial if $\phi(x y)=\phi(y x)$ for all $x, y \in \mathcal{A}$. Assume that $\mathcal{A}$ is a $W^{*}$-probability space, $\phi$ is faithful if $\phi\left(x x^{*}\right)=0$ if and only if $x=0, \phi$ is non-degenerated if the GNS representation associated with $\phi$ is faithful. The elements in $\mathcal{A}$ are called noncommutative random variables. Let $x \in \mathcal{A}$ be a random variable, the distribution of $x$ is a linear functional $\mu_{x}$ on $\mathbb{C}[X]$ such that

$$
\mu_{x}(P)=\phi(P(x))
$$

for all $P \in \mathbb{C}[X]$, where $\mathbb{C}[X]$ is the set of complex-coefficient polynomials in one variable.

Remark 2.2. In this paper, the commutative probability space $\mathcal{A}$ is assumed to be set of all complex-valued random variables from $(\Omega, \mu)$, where $\Omega$ is a classical probability space. It is well know that $\mathcal{A}$ is an algebra and $\phi(\cdot)=\int \cdot d \mu$ defines a linear functional on $\bigcap_{1 \leq p<\infty} L^{p}(\Omega)$. In this case, the supports of random variables are not necessarily compact. When $(\mathcal{A}, \phi)$ is a $W^{*}$ probability space where $\phi$ is faithful and tracial, we can also consider non-compact supported noncommutative random variables.

Definition 2.3. Let $I$ be an index set. The algebra of noncommutative polynomials in $|I|$ variables, $\mathbb{C}\left\langle X_{i} \mid i \in I\right\rangle$, is the linear span of 1 and noncommutative monomials of the form $X_{i_{1}}^{k_{1}} X_{i_{2}}^{k_{2}} \cdots X_{i_{n}}^{k_{n}}$ with $i_{1} \neq i_{2} \neq \cdots \neq i_{n} \in I$ and all $k_{j}$ 's are positive integers. For convenience, we denote by $\mathbb{C}\left\langle X_{i} \mid i \in I\right\rangle_{0}$ the set of noncommutative polynomials without a constant term. Let $\left(x_{i}\right)_{i \in I}$ be a family of random variables in a noncommutative probability space $(\mathcal{A}, \phi)$. Their joint distribution is a linear functional $\mu: \mathbb{C}\left\langle X_{i} \mid i \in I\right\rangle \rightarrow \mathbb{C}$ defined by

$$
\mu\left(X_{i_{1}}^{k_{1}} X_{i_{2}}^{k_{2}} \cdots X_{i_{n}}^{k_{n}}\right)=\phi\left(x_{i_{1}}^{k_{1}} x_{i_{2}}^{k_{2}} \cdots x_{i_{n}}^{k_{n}}\right),
$$

and $\mu(1)=1$.

Remark 2.4. The joint distribution of random variables depends on their order. For example, when $I=\{1,2\}$, then $\mu_{x_{1}, x_{2}}$ may not equal $\mu_{x_{2}, x_{1}}$. According to our notation, $\mu_{x_{1}, x_{2}}\left(X_{1} X_{2}\right)=$ $\phi\left(x_{1} x_{2}\right)$, but $\mu_{x_{2}, x_{1}}\left(X_{1} X_{2}\right)=\phi\left(x_{2} x_{1}\right)$.

Now, we turn to define operator-valued probability spaces which is a generalization of scalarvalued noncommutative probability spaces.

Definition 2.5. An operator valued probability space $(\mathcal{A}, \mathcal{B}, \mathbb{E}: \mathcal{A} \rightarrow \mathcal{B})$ consists of an algebra $\mathcal{A}$, a subalgebra $\mathcal{B}$ of $\mathcal{A}$ and a $\mathcal{B}-\mathcal{B}$ bimodule linear map $\mathbb{E}: \mathcal{A} \rightarrow \mathcal{B}$ i.e.

$$
\mathbb{E}\left[b_{1} a b_{2}\right]=b_{1} \mathbb{E}[a] b_{2}, \quad \mathbb{E}[b]=b
$$

for all $b_{1}, b_{2}, b \in \mathcal{B}$ and $a \in \mathcal{A}$. According to the definition in [21], we call $\mathbb{E}$ a conditional expectation from $\mathcal{A}$ to $\mathcal{B}$ if $\mathbb{E}$ is onto, i.e. $\mathbb{E}[\mathcal{A}]=\mathcal{B}$. The elements of $\mathcal{A}$ are called $\mathcal{B}$-valued random variables or random variables for simple.

Definition 2.6. Given an algebra $\mathcal{B}$, we denote by $\mathcal{B}\langle X\rangle$ the algebra which is freely generated by $\mathcal{B}$ and the indeterminant $X$. Let $1_{X}$ be the identity of $\mathcal{B}\langle X\rangle$, then $\mathcal{B}\langle X\rangle$ is set of linear combinations of the elements in $\mathcal{B}$ and the noncommutative monomials $b_{0} X b_{1} X b_{2} \cdots b_{n-1} X b_{n}$ where $b_{k} \in \mathcal{B} \cup\left\{\mathbb{C} 1_{X}\right\}$ and $n \geq 0$. The elements in $\mathcal{B}\langle X\rangle$ are called $\mathcal{B}$-polynomials. In addition, 
$\mathcal{B}\langle X\rangle_{0}$ denotes the subalgebra of $\mathcal{B}\langle X\rangle$ which does not contain a constant term, i.e. the linear span of the noncommutative monomials $b_{0} X b_{1} X b_{2} \cdots b_{n-1} X b_{n}$ where $b_{k} \in \mathcal{B} \cup\left\{\mathbb{C} 1_{X}\right\}$ and $n \geq 1$.

Operator-valued independence relations are defined as follows:

Definition 2.7. Given an operator valued probability space $(\mathcal{A}, \mathcal{B}, \mathbb{E}: \mathcal{A} \rightarrow \mathcal{B})$ such that $\mathcal{A}$ and $\mathcal{B}$ are unital.

- Suppose that $\mathcal{A}$ is commutative. A family of unital subalgebras $\left\{\mathcal{A}_{i} \supset \mathcal{B}\right\}_{i \in I}$ are said to be conditionally independent with respect to $\mathbb{E}$ if

$$
\mathbb{E}\left[a_{1} \cdots a_{n}\right]=\mathbb{E}\left[a_{1}\right] \mathbb{E}\left[a_{2}\right] \cdots \mathbb{E}\left[a_{n}\right],
$$

whenever $a_{k} \in \mathcal{A}_{i_{k}}$ and $i_{1}, \ldots, i_{n}$ are pairwisely different. A family of random variables $\left(x_{i}\right)_{i \in I}$ are said to be conditionally independent over $\mathcal{B}$ if the unital subalgebras $\left\{\mathcal{A}_{i}\right\}_{i \in I}$ which are generated by $x_{i}$ and $\mathcal{B}$ respectively are conditionally independent, or equivalently

$$
\mathbb{E}\left[p_{1}\left(x_{i_{1}}\right) p_{2}\left(x_{i_{2}}\right) \cdots p_{n}\left(x_{i_{n}}\right)\right]=\mathbb{E}\left[p_{1}\left(x_{i_{1}}\right)\right] \mathbb{E}\left[p_{2}\left(x_{i_{2}}\right)\right] \cdots \mathbb{E}\left[p_{n}\left(x_{i_{n}}\right)\right],
$$

whenever $i_{1}, \ldots, i_{n}$ are pairwisely different and $p_{1}, \ldots, p_{n} \in \mathcal{B}\langle X\rangle$.

- A family of unital subalgebras $\left\{\mathcal{A}_{i} \supset \mathcal{B}\right\}_{i \in I}$ are said to be freely independent with respect to $\mathbb{E}$ if

$$
\mathbb{E}\left[a_{1} \cdots a_{n}\right]=0
$$

whenever $i_{1} \neq i_{2} \neq \cdots \neq i_{n}, a_{k} \in \mathcal{A}_{i_{k}}$ and $\mathbb{E}\left[a_{k}\right]=0$ for all $k$. A family of random variables $\left(x_{i}\right)_{i \in I}$ are said to be freely independent over $\mathcal{B}$, if the unital subalgebras $\left\{\mathcal{A}_{i}\right\}_{i \in I}$ which are generated by $x_{i}$ and $B$ respectively are freely independent, or equivalently

$$
\mathbb{E}\left[p_{1}\left(x_{i_{1}}\right) p_{2}\left(x_{i_{2}}\right) \cdots p_{n}\left(x_{i_{n}}\right)\right]=0,
$$

whenever $i_{1} \neq i_{2} \neq \cdots \neq i_{n}, p_{1}, \ldots, p_{n} \in \mathcal{B}\langle X\rangle$ and $\mathbb{E}\left[p_{k}\left(x_{i_{k}}\right)\right]=0$ for all $k$.

- A family of unital subalgebras $\left\{\mathcal{A}_{i} \supset \mathcal{B}\right\}_{i \in I}$ are said to be Boolean independent with respect to $\mathbb{E}$ if

$$
\mathbb{E}\left[a_{1} \cdots a_{n}\right]=\mathbb{E}\left[a_{1}\right] \mathbb{E}\left[a_{2}\right] \cdots \mathbb{E}\left[a_{n}\right]
$$

whenever $a_{k} \in \mathcal{A}_{i_{k}}$ and $i_{1} \neq i_{2} \neq \cdots \neq i_{n}$. A family of random variables $\left(x_{i}\right)_{i \in I}$ are said to be Boolean independent over $\mathcal{B}$, if the non-unital subalgebras $\left\{\mathcal{A}_{i}\right\}_{i \in I}$ which are generated by $x_{i}$ and $B$ respectively are Boolean independent, or equivalently

$$
\mathbb{E}\left[p_{1}\left(x_{i_{1}}\right) p_{2}\left(x_{i_{2}}\right) \cdots p_{n}\left(x_{i_{n}}\right)\right]=\mathbb{E}\left[p_{1}\left(x_{i_{1}}\right)\right] \mathbb{E}\left[p_{2}\left(x_{i_{2}}\right)\right] \cdots \mathbb{E}\left[p_{n}\left(x_{i_{n}}\right)\right],
$$

whenever $i_{1} \neq i_{2} \neq \cdots \neq i_{n}$ and $p_{1}, \ldots, p_{n} \in \mathcal{B}\langle X\rangle_{0}$.

Remark 2.8. When $\mathcal{B}=\mathbb{C}$ in the above definition, we get the independence relations for scalar-valued probability.

2.2. Partitions and cumulants. In this subsection, we recall some combinatorial tools for classical, free and Boolean independence. See [1, 11, 19] for more details.

Definition 2.9. Let $S$ be an ordered set:

1. A partition $\pi$ of a set $S$ is a collection of disjoint, nonempty sets $V_{1}, \ldots, V_{r}$ whose union is $S$. $V_{1}, \ldots, V_{r}$ are called blocks of $\pi$. The collection of all partitions of $S$ will be denoted by $P(S)$.

2. Given two partitions $\pi, \sigma$, we say $\pi \leq \sigma$ if each block of $\pi$ is contained in a block of $\sigma$. 
3. A partition $\pi \in P(S)$ is noncrossing if there is no quadruple $\left(s_{1}, s_{2}, r_{1}, r_{2}\right)$ such that $s_{1}<r_{1}<s_{2}<r_{2}, s_{1}, s_{2} \in V, r_{1}, r_{2} \in W$ and $V, W$ are two different blocks of $\pi$.

4. A partition $\pi \in P(S)$ is interval if there is no triple $\left(s_{1}, s_{2}, r\right)$ such that $s_{1}<r<s_{2}$, $s_{1}, s_{2} \in V, r \in W$ and $V, W$ are two different blocks of $\pi$.

5. Let $\mathbf{i}=\left(i_{1}, \ldots, i_{k}\right)$ be a sequence of indices of $I$ and $[k]=\{1, \ldots, k\}$. We denote by ker $\mathbf{i}$ the element of $P([k])$ whose blocks are the equivalence classes of the relation

$$
s \sim t \Leftrightarrow i_{s}=i_{t}
$$

Remark 2.10. It is obvious that interval partitions are noncrossing.

Definition 2.11. Let $(\mathcal{A}, \mathcal{B}, \mathbb{E}: \mathcal{A} \rightarrow \mathcal{B})$ be an operator valued probability space:

1. A $\mathcal{B}$-functional is a $n$-linear map $\rho: \mathcal{A}^{n} \rightarrow \mathcal{B}$ such that

$$
\rho\left(b_{0} a_{1} b_{1}, a_{2} b_{2}, \ldots, a_{n} b_{n}\right)=b_{0} \rho\left(a_{1}, b_{1} a_{2}, \ldots, b_{n-1} a_{n}\right) b_{n}
$$

for all $b_{0}, \ldots, b_{n} \in \mathcal{B} \cup\left\{1_{A}\right\}$.

2. For $k \in \mathbb{N}$, let $\rho^{(k)}$ be a $\mathcal{B}$-functional from $\mathcal{A}^{k}$ to $\mathcal{B}$. Suppose that $\mathcal{A}$ is commutative. Given $\pi \in P(n)$, we can define a $\mathcal{B}$-functional $\rho^{(\pi)}: \mathcal{A}^{n} \rightarrow \mathcal{B}$ by the formula:

$$
\rho^{(\pi)}\left(a_{1}, \ldots, a_{n}\right)=\prod_{V \in \pi} \rho(V)\left(a_{1}, \ldots, a_{n}\right),
$$

where if $V=\left(i_{1}<i_{2}<\cdots<i_{s}\right)$ is a block of $\pi$ then

$$
\rho(V)\left(a_{1}, \ldots, a_{n}\right)=\rho^{(s)}\left(a_{i_{1}}, \ldots, a_{i_{s}}\right) .
$$

If $\mathcal{A}$ is not commutative, then there is no natural way to define $\rho^{(\pi)}\left(a_{1}, \ldots, a_{n}\right)$ for $\pi \notin$ $N C(n)$. For $\pi \in N C(n)$, the $\mathcal{B}$-functional $\rho^{(\pi)}: \mathcal{A}^{n} \rightarrow \mathcal{B}$ can be defined recursively as follows:

$$
\rho^{(\pi)}\left(a_{1}, \ldots, a_{n}\right)=\rho^{(\pi \backslash V)}\left(a_{1}, \ldots, a_{l} \rho^{(s)}\left(a_{l+1, \ldots, a_{l+s}}\right), a_{l+s+1}, \ldots, a_{n}\right)
$$

where $V=(l+1, l+2, \ldots, l+s)$ is an interval block of $\pi$.

Definition 2.12. Let $(\mathcal{A}, \mathcal{B}, \mathbb{E}: \mathcal{A} \rightarrow \mathcal{B})$ be an operator-valued probability space:

1. If $\mathcal{A}$ is commutative, then the operator-valued classical cumulants $c_{\mathbb{E}}^{(n)}: \mathcal{A}^{n} \rightarrow \mathcal{B}$ are defined by the classical moment-cumulant formula:

$$
\mathbb{E}\left[a_{1} \cdots a_{n}\right]=\sum_{\pi \in P(n)} c_{\mathbb{E}}^{(\pi)}\left(a_{1}, \ldots, a_{n}\right),
$$

for all $a_{1}, \ldots, a_{n} \in \mathcal{A}$.

2. The operator-valued free cumulants $\kappa_{\mathbb{E}}^{(k)}: \mathcal{A}^{n} \rightarrow \mathcal{B}$ are defined by the free momentcumulant formula:

$$
\mathbb{E}\left[a_{1} \cdots a_{n}\right]=\sum_{\pi \in N C(n)} \kappa_{\mathbb{E}}^{(\pi)}\left(a_{1}, \ldots, a_{n}\right),
$$

for all $a_{1}, \ldots, a_{n} \in \mathcal{A}$.

3. The operator-valued Boolean cumulants $b_{\mathbb{E}}^{(k)}: \mathcal{A}^{n} \rightarrow \mathcal{B}$ are defined by the Boolean moment-cumulant formula:

$$
\mathbb{E}\left[a_{1} \cdots a_{n}\right]=\sum_{\pi \in I(n)} b_{\mathbb{E}}^{(\pi)}\left(a_{1}, \ldots, a_{n}\right),
$$

for all $a_{1}, \ldots, a_{n} \in \mathcal{A}$. 
Note that all these three types of cumulants can be resolved recursively, e.g.

$$
c_{\mathbb{E}}^{(1)}\left(a_{1}\right)=\mathbb{E}\left[a_{1}\right]
$$

and

$$
c_{\mathbb{E}}^{(n)}\left(a_{1}, \ldots, a_{n}\right)=\mathbb{E}\left[a_{1} \cdots a_{n}\right]-\sum_{\pi \in P(n), \pi \neq 1_{n}} c_{\mathbb{E}}^{(\pi)}\left(a_{1}, \ldots, a_{n}\right),
$$

where $c_{\mathbb{E}}^{(\pi)}\left(a_{1}, \ldots, a_{n}\right)$ depends on $c_{\mathbb{E}}^{(k)}\left(a_{1}, \ldots, a_{n}\right)$ for $k=1, \ldots, n-1$ if $\pi \neq 1_{n}$. Similarly, we determine $\kappa_{\mathbb{E}}^{(n)}$ and $b_{\mathbb{E}}^{(n)}$ by substituting $P(n)$ for $N C(n)$ and $I(n)$, respectively.

See [2] and [14, we have the following vanishing-cumulant conditions for independence relations.

Theorem 2.13. Let $(\mathcal{A}, \mathcal{B}, \mathbb{E}: \mathcal{A} \rightarrow \mathcal{B})$ be an operator-valued probability space and $\left(x_{i}\right)_{i \in I}$ be a family of random variables in $\mathcal{A}$ :

1. If $\mathcal{A}$ is is commutative, then $\left(x_{i}\right)_{i \in I}$ are conditionally independent with respect to $\mathbb{E}$ iff

$$
c_{\mathbb{E}}^{(n)}\left(b_{0} x_{i_{1}} b_{1}, \ldots, x_{i_{n}} b_{n}\right)=0,
$$

whenever $i_{k} \neq i_{l}$ for some $1 \leq k, l \leq n$.

2. $\left(x_{i}\right)_{i \in I}$ are free independent with respect to $\mathbb{E}$ iff

$$
\kappa_{\mathbb{E}}^{(n)}\left(b_{0} x_{i_{1}} b_{1}, \ldots, x_{i_{n}} b_{n}\right)=0,
$$

whenever $i_{k} \neq i_{l}$ for some $1 \leq k, l \leq n$.

3. $\left(x_{i}\right)_{i \in I}$ are Boolean independent with respect to $\mathbb{E}$ iff

$$
b_{\mathbb{E}}^{(n)}\left(b_{0} x_{i_{1}} b_{1}, \ldots, x_{i_{n}} b_{n}\right)=0,
$$

whenever $i_{k} \neq i_{l}$ for some $1 \leq k, l \leq n$.

The following theorem gives combinatorial characterizations for joint distributions of classical, free and Boolean independence. For classical and free independence, see [2]. For Boolean independence, see [7, 14].

Theorem-Definition 2.14. Let $(\mathcal{A}, \mathcal{B}, \mathbb{E}: \mathcal{A} \rightarrow \mathcal{B})$ be an operator-valued probability space, and $\left(x_{i}\right)_{i \in I}$ be a family of random variables in $\mathcal{A}$ :

1. If $\mathcal{A}$ is is commutative, then the $\mathcal{B}$-valued joint distribution of $\left(x_{i}\right)_{i \in I}$ has the property corresponding to $D$ in the table below iff for any $\pi \in P(n)$.

$$
c_{\mathbb{E}}^{(\pi)}\left(b_{0} x_{i_{1}} b_{1}, \ldots, x_{i_{n}} b_{n}\right)=0,
$$

unless $\pi \in D(n)$ and $\pi \leq$ keri, where $\mathbf{i}=\left(i_{1}, \ldots, i_{n}\right)$.

\begin{tabular}{|ll|}
\hline Partitions D & Joint distribution \\
\hline$P:$ All partitions & Classical independent \\
$P_{h}:$ Partitions with even block sizes & Classical independent and symmetric \\
$P_{b}:$ Partitions with block size 1 or 2 & Classical independent and Gaussian \\
$P_{2}:$ Pair partitions & Classical independent and centered Gaussian \\
\hline
\end{tabular}

2. The $\mathcal{B}$-valued joint distribution of $\left(x_{i}\right)_{i \in I}$ has the property corresponding to $D$ in the table below iff for any $\pi \in P(n)$.

$$
\kappa_{\mathbb{E}}^{(\pi)}\left(b_{0} x_{i_{1}} b_{1}, \ldots, x_{i_{n}} b_{n}\right)=0
$$

unless $\pi \in D(n)$ and $\pi \leq$ keri $\mathbf{i}$, where $\mathbf{i}=\left(i_{1}, \ldots, i_{n}\right)$. 


\begin{tabular}{|ll|}
\hline Partitions D & Joint distribution \\
\hline$P:$ Noncrossing partitions & Free independent \\
$P_{h}:$ Noncrossing Partitions with even block sizes & Free independent and symmetric \\
$P_{b}:$ Noncrossing Partitions with block size 1 or 2 & Free independent and semicircular \\
$P_{2}:$ Noncrossing Pair partitions & Free independent and centered semicircular \\
\hline
\end{tabular}

3. The $\mathcal{B}$-valued joint distribution of $\left(x_{i}\right)_{i \in I}$ has the property corresponding to $D$ in the table below iff for any $\pi \in P(n)$.

$$
b_{\mathbb{E}}^{(\pi)}\left(b_{0} x_{i_{1}} b_{1}, \ldots, x_{i_{n}} b_{n}\right)=0,
$$

unless $\pi \in D(n)$ and $\pi \leq k e r \mathbf{i}$, where $\mathbf{i}=\left(i_{1}, \ldots, i_{n}\right)$.

\begin{tabular}{|ll|}
\hline Partitions D & Joint distribution \\
\hline$I:$ Interval partitions & Boolean independent \\
$I_{h}:$ Interval partitions with even block sizes & Boolean independent and symmetric \\
$I_{b}:$ Interval partitions with block size 1 or 2 & Boolean independent and Bernoulli \\
$I_{2}:$ Interval pair partitions & Boolean independent and centered Bernoulli \\
\hline
\end{tabular}

Remark 2.15. Given an operator-valued probability space $(\mathcal{A}, \mathcal{B}, \mathbb{E})$, a random variable $x$ is symmetrically distributed if and only if

$$
\mathbb{E}\left[b_{0} x b_{1} \cdots x b_{n}\right]=0,
$$

whenever $n$ is odd.

Remark 2.16. Nonzero classical operator-valued centered Gaussian random variables are unbounded. Let $k$ be a natural number. It is well known that $\left|P_{2}(2 k)\right|=\frac{(2 k) !}{2^{k} k !}$. Let $x$ be a nonzero Gaussian random variable from $(\mathcal{A}, \mathcal{B}, \mathbb{E})$. Then we have $a=c_{\mathbb{E}}^{(2)}(x, x)=\mathbb{E}\left[x^{2}\right] \neq 0$ and $\mathbb{E}\left[x^{2 k}\right]=\frac{(2 k) !}{2^{k} k !} a^{k}$. Therefore,

$$
\|x\| \geq\left\|\mathbb{E}\left[x^{2 k}\right]\right\|^{1 / 2 k}=\left\|\frac{(2 k) !}{2^{k} k !} a^{k}\right\|^{1 / 2 k}=\left(\frac{(2 k) !}{2^{k} k !}\right)^{1 / 2 k}\|a\|^{1 / 2} \rightarrow \infty, \quad k \rightarrow \infty .
$$

Therefore, to include Gaussian random variables, we must take account of unbounded random variables.

\section{Noncommutative Distributional SYMmetries}

In this section, we will recall distributional symmetries for classical and free independence. See [4] for more details.

Definition 3.1. An orthogonal Hopf algebra is a unital $C^{*}$-algebra $A$ generated by $n^{2}$ selfadjoint elements $\left\{u_{i, j} \mid i, j=1, \ldots, n\right\}$, such that the following hold:

1. The inverse of $u=\left(u_{i, j}\right)_{i, j=1, \ldots . n} \in M_{n}(A)$ is the transpose $u^{t}=\left(u_{j, i}\right)_{i, j=1, \ldots n}$, i.e. $\sum_{k=1}^{n} u_{i, k} u_{j, k}=\sum_{k=1}^{n} u_{k, i} u_{k, j}=\delta_{i, j} 1_{A}$.

2. $\Delta\left(u_{i, j}\right)=\sum_{k=1}^{n} u_{i, k} \otimes u_{k, j}$ determines a $C^{*}$-unital homomorphism $\Delta: A \rightarrow A \otimes_{\min } A$.

3. $\epsilon\left(u_{i, j}\right)=\delta_{i, j}$ defines a homomorphism $\epsilon: A \rightarrow \mathbb{C}$.

4. $S\left(u_{i, j}\right)=u_{j, i}$ defines a homomorphism $S: A \rightarrow A^{o p}$. 
Following the notion of Wang's free quantum groups in [23, 24], one can define universal algebras $A$ generated by $n^{2}$ noncommutative variables $\left\{u_{i, j}\right\}_{i, j=1, \ldots, n}$ which satisfy some relations $R$. Moreover, for suitable choices of $R$, we will get Hopf algebras in the sense of Woronowicz [26].

In [4, Banica and Speicher found the following conditions for defining Hopf orthogonal algebras.

Definition 3.2. A matrix $u=\left(u_{i, j}\right)_{i, j=1, \ldots, n} \in M_{n}(A)$ over a $C^{*}$-algebra $A$ is said to be:

- Orthogonal, if all entries of $u$ are selfadjoint, and $u u^{t}=u^{t} u=1_{n}$, where $u^{t}$ the transpose of $u$.

- Magic, if it is orthogonal and its entries are projections.

- Cubic, if it is orthogonal and $u_{i, j} u_{i, k}=u_{j, i} u_{k, i}=0$, for $j \neq k$.

- Bistochastic, if it is orthogonal and $\sum_{i=1}^{n} u_{i, j}=\sum_{j=1}^{n} u_{k, i}=1_{A}$, for all $j, k$.

- Magic', if it is cubic with the same sum on rows and columns.

- Bistochastic', if it is orthogonal with the same sum on rows and columns.

The universal algebras associated with the above conditions are defined as follows:

Definition 3.3. $A_{g}(n)$ with $g=o, s, h, b, s^{\prime}, b^{\prime}$ is the universal $C^{*}$-algebra generated by the entries of an $n \times n$ matrix which is respectively orthogonal, magic, cubic, bistochastic, magic' and bistochastic'. $C_{g}(n)$ with $g=o, s, h, b, s^{\prime}, b^{\prime}$ is the universal commutative $C^{*}$-algebra generated by the entries of an $n \times n$ matrix which is respectively orthogonal, magic, cubic, bistochastic, magic' and bistochastic'.

Especially, for each $n, A_{s}(n)$ and $A_{o}(n)$ are Wang's quantum permutation group and quantum orthogonal group, respectively [24, 23]. $C_{g}(n)$ can be considered as the abelianization of $A_{g}(n)$ for $g=o, s, h, b, s^{\prime}, b^{\prime}$. It should be mentioned here that there are 7 easy quantum groups in total, see [25].

Given two orthogonal Hopf algebras $(A, u)$ and $(B, v)$ such that $A$ is generated by $u=$ $\left\{u_{i, j} \mid i, j=1, \ldots n\right\}$ and $B$ is generated by $v=\left\{v_{i, j} \mid i, j=1, \ldots n\right\}$. We denote by $(A, u) \rightarrow(B, v)$ if there exists a $C^{*}$-homomorphism $\eta$ from $A$ to $B$ such that $\eta\left(u_{i, j}\right)=v_{i, j}$. In other words, $(A, u) \rightarrow(B, v)$ implies that $B$ is a quotient $C^{*}$-algebra of $A$. Then, for each $n$, we have the following diagrams:

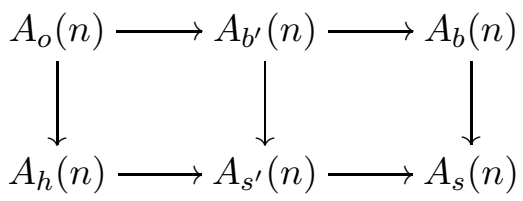

and

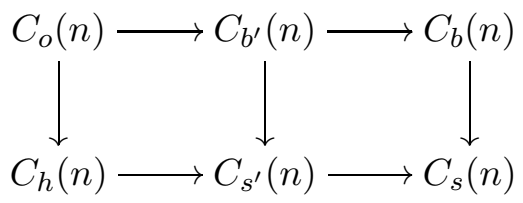

and

$$
A_{g}(n) \rightarrow C_{g}(n)
$$

for $g=o, s, h, b, s^{\prime}, b^{\prime}$.

For convenience, we will denote by $B \subseteq A$ the relation $(A, u) \rightarrow(B, v)$. 
Proposition 3.4. Let $E(n)$ be an orthogonal Hopf algebra generated by $n^{2}$ selfadjoint elements $\left\{u_{i, j}\right\}_{i, j=1, \ldots, n}$. Then, we have the following.

1. If $E(n) \not \subset A_{h}(n)$, then there exists an index $j$ such that $\sum_{k=1}^{n} u_{k, j}^{4} \neq 1_{E(n)}$.

2. If $E(n) \not \subset A_{b}(n)$, then there exists an index $j$ such that $\sum_{k=1}^{n} u_{k, j} \neq 1_{E(n)}$.

Proof. 1. Suppose $\sum_{k=1}^{n} u_{k, i}^{4}=1_{E(n)}$, for all $i$. Since $\sum_{k=1}^{n} u_{k, i}^{2}=1_{E(n)}$ and $u_{k, i}^{4} \leq u_{k, i}^{2}$, we have

$$
u_{k, i}^{4}=u_{k, i}^{2}
$$

$\left(u_{i, j}^{2}\right)_{i, j=1, \ldots, n}$ is a matrix of orthogonal projections with sum 1 on rows and columns. Therefore,

$$
u_{i, j}^{2} u_{i, k}^{2}=u_{j, i}^{2} u_{k, i}^{2}=0,
$$

for $j \neq k$. Since $u_{i, j}$ and $u_{i, k}$ are selfadjoint, we have

$$
u_{i, j} u_{i, k}=u_{j, i} u_{k, i}=0 .
$$

It implies that $E(n)$ is a quotient algebra of $A_{h}(n)$, which is a contradiction.

2. Suppose $\sum_{k=1}^{n} u_{k, i}=1_{E(n)}$, for all $i$. Then, for each $i$, we have

$$
\sum_{l=1}^{n} u_{i, l}=\sum_{l=1}^{n} \sum_{k=1}^{n} u_{i, l} u_{k, l}=\sum_{k=1}^{n} \sum_{l=1}^{n} u_{i, l} u_{k, l}=\sum_{k=1}^{n} \delta_{i, k} 1_{E(n)}=1_{E(n) .}
$$

Therefore, $E(n)$ is a quotient algebra of $A_{b}(n)$, which is a contradiction.

Proposition 3.5. Let $E(n)$ be an orthogonal Hopf algebra generated by $n^{2}$ selfadjoint elements $\left\{u_{i, j}\right\}_{i, j=1, \ldots, n}$ such that $A_{s}(n) \subseteq E(n) \subseteq A_{o}(n)$. Then, the following hold:

1. If $E(n) \subseteq A_{h}(n)$ and $E(n) \subseteq A_{b}(n)$, then $E(n)=A_{s}(n)$.

2. If $E(n) \nsubseteq A_{h}(n)$ and $E(n) \subseteq A_{b}(n)$, then $\exists i^{\prime}$ such that

$$
\sum_{k=1}^{n} u_{k, i^{\prime}}^{m} \neq 1
$$

for all $m>2$.

3. If $E(n) \nsubseteq A_{b}(n)$ and $E(n) \subseteq A_{h}(n)$, then $\exists i^{\prime}$ such that

$$
\sum_{k=1}^{n} u_{k, i^{\prime}}^{m} \neq 1
$$

for all odd numbers $m$.

4. If $E(n) \nsubseteq A_{h}(n)$ and $E(n) \nsubseteq A_{b}(n)$, then $\exists i_{1}^{\prime}, i_{2}^{\prime}$ such that

$$
\sum_{k=1}^{n} u_{k, i_{1}^{\prime}}^{m} \neq 1
$$

for all $m>2$, and

$$
\sum_{k=1}^{n} u_{k, i_{2}^{\prime}} \neq 1
$$


Proof. It is obvious that $\left\|u_{i, j}\right\| \leq 1$ for all $i, j=1, \ldots n$.

1. By assumption, we have

$$
\sum_{k=1}^{n} u_{i, k}=1_{E(n)}
$$

and

for $j \neq k$. Therefore,

$$
u_{i, j} u_{i, k}=0
$$

$$
u_{i, j}=u_{i, j} \sum_{k=1}^{n} u_{i, k}=u_{i, j}^{2}
$$

for all $i, j$. It implies that $E(n)$ is a quotient algebra of $A_{s}(n)$, so $E(n)=A_{s}(n)$.

2. By Proposition 3.4 there exists $i^{\prime}$ such that

$$
\sum_{k=1}^{n} u_{k, i^{\prime}}^{4} \neq 1 \text {. }
$$

Therefore, there exists $k^{\prime}$ such that

$$
u_{k^{\prime}, i^{\prime}}^{4}<u_{k^{\prime}, i^{\prime}}^{2}
$$

which implies that the spectrum of $u_{k^{\prime}, i^{\prime}}$ contains $a$ such that $-1<a<1$. Therefore,

$$
u_{k^{\prime}, i^{\prime}}^{m}<u_{k^{\prime}, i^{\prime}}^{2}
$$

for all natural number $m>2$. Hence, we have

$$
\sum_{k=1}^{n} u_{k, i^{\prime}}^{m}<1_{E(n)},
$$

for $m>2$.

3. According to Proposition 3.4, there exists $i^{\prime}$ such that

$$
\sum_{k=1}^{n} u_{k, i^{\prime}} \neq 1
$$

Therefore, there exists $k^{\prime}$ such that $u_{k^{\prime}, i^{\prime}}$ is not an orthogonal projection which implies that

$$
u_{k^{\prime}, i^{\prime}}^{2 m+1}<u_{k^{\prime}, i^{\prime}}^{2 m}
$$

Thus, we have

$$
\sum_{k=1}^{n} u_{k, i^{\prime}}^{2 m+1}<\sum_{k=1}^{n} u_{k, i^{\prime}}^{2 m}=1_{E(n)},
$$

4. Combine Case 2 and 3, the proof is complete.

Following the proof above, we have following properties for abelian orthogonal Hopf algebras.

Corollary 3.6. Let $E(n)$ be an orthogonal Hopf algebra generated by $n^{2}$ selfadjoint elements $\left\{u_{i, j}\right\}_{i, j=1, \ldots, n}$ such that $C_{s}(n) \subseteq E(n) \subseteq C_{o}(n)$. Then, the following hold:

1. If $E(n) \subseteq C_{h}(n)$ and $E(n) \subseteq C_{b}(n)$, then $E(n)=C_{s}(n)$.

2. If $E(n) \nsubseteq C_{h}(n)$ and $E(n) \subseteq C_{b}(n)$, then there exists $i^{\prime}$ such that $\sum_{k=1}^{n} u_{k, i^{\prime}}^{m} \neq 1$, for all $m>2$. 
3. If $E(n) \nsubseteq C_{b}(n)$ and $E(n) \subseteq C_{h}(n)$, then there exists $i^{\prime}$ such that $\sum_{k=1}^{n} u_{k, i^{\prime}}^{m} \neq 1$, for all odd numbers $m$.

4. If $E(n) \nsubseteq C_{h}(n)$ and $E(n) \nsubseteq C_{b}(n)$, then there exist $i_{1}^{\prime}, i_{2}$ such that $\sum_{k=1}^{n} u_{k, i_{1}^{\prime}}^{m} \neq 1$, for all $m>2$, and $\sum_{k=1}^{n} u_{k, i_{2}^{\prime}} \neq 1$.

Now, we turn to define noncommutative distributional symmetries by maps of quantum family in the sense of Soltan [17].

Definition 3.7. Let $(A, \Delta)$ be a quantum group and $\mathcal{V}$ be a unital algebra. By a (right) coaction of the quantum group $A$ on $\mathcal{V}$, we mean a unital homomorphism $\alpha: \mathcal{V} \rightarrow \mathcal{V} \otimes \mathcal{A}$ such that

$$
\left(\alpha \otimes i d_{A}\right) \alpha=(i d \otimes \Delta) \alpha .
$$

Definition 3.8. Given an orthogonal Hopf algebra $E(n)$ generated by $\left\{u_{i, j}\right\}_{i, j=1, \ldots n}$, we have a natural coaction $\alpha_{n}$ of $E(n)$ on $\mathbb{C}\left\langle X_{1}, \ldots, X_{n}\right\rangle$ such that

$$
\alpha_{n}: \mathbb{C}\left\langle X_{1}, \ldots, X_{n}\right\rangle \rightarrow \mathbb{C}\left\langle X_{1}, \ldots, X_{n}\right\rangle \otimes E(n)
$$

is an algebraic homomorphism defined via $\alpha_{n}\left(X_{i}\right)=\sum_{k=1}^{n} X_{k} \otimes u_{k, i}$ for all $i=1, \ldots, n$.

Definition 3.9. Given a probability space $(\mathcal{A}, \phi)$, a sequence of random variables $\left(x_{1}, \ldots, x_{n}\right)$ of $\mathcal{A}$ and an orthogonal Hopf algebra $E(n)$ generated by $\left\{u_{i, j}\right\}_{i, j=1, \ldots . n}$. We say that the joint distribution $\mu_{x_{1}, \ldots, x_{n}}$ of $x_{1}, \ldots, x_{n}$ is $E(n)$-invariant if

$$
\mu_{x_{1}, \ldots, x_{n}}(p) 1_{E(n)}=\mu_{x_{1}, \ldots, x_{n}} \otimes i d_{E(n)}\left(\alpha_{n}(p)\right),
$$

for all $p \in \mathbb{C}\left\langle X_{1}, \ldots, X_{n}\right\rangle$.

Proposition 3.10. Given a probability space $(\mathcal{A}, \phi)$ and a sequence of random variables $\left(x_{1}, \ldots, x_{n}\right)$ of $\mathcal{A}$. $E_{1}(n)$ and $E_{2}(n)$ are two orthogonal Hopf algebras such that $E_{1}(n) \subseteq E_{2}(n)$. Then, $\left(x_{1}, \ldots, x_{n}\right)$ is $E_{1}(n)$-invariant if $E_{2}(n)$-invariant.

Proof. Let $\left\{u_{i, j}^{(l)}\right\}_{i, j=1, \ldots, n}$ be generators of $E_{l}(n)$ for $l=1,2$. Since $E_{1}(n) \subseteq E_{2}(n)$, there exists a $C^{*}$-homomorphism $\Phi: E_{2}(n) \rightarrow E_{1}(n)$ such that

$$
\Phi\left(u_{i, j}^{(2)}\right)=u_{i, j}^{(1)}
$$

for all $i, j .\left(x_{1}, \ldots, x_{n}\right)$ is $E_{2}(n)$-invariant is equivalent to that

$$
\mu_{x_{1}, \ldots, x_{n}}\left(X_{\mathbf{i}}\right) 1_{E_{2}(n)}=\sum_{\mathbf{j} \in[n]^{k}} \mu_{x_{1}, \ldots, x_{n}}\left(X_{\mathbf{j}}\right) \otimes u_{\mathbf{i}, \mathbf{j}}^{(2)},
$$

for all monomials $X_{i_{1}} \cdots X_{i_{k}} \in \mathbb{C}\left\langle X_{1}, \ldots, X_{n}\right\rangle$. Apply $\Phi$ on both sides of the above equation, we get

$$
\mu_{x_{1}, \ldots, x_{n}}\left(X_{\mathbf{i}}\right) 1_{E_{1}(n)}=\sum_{\mathbf{j} \in[n]^{k}} \mu_{x_{1}, \ldots, x_{n}}\left(X_{\mathbf{j}}\right) \otimes u_{\mathbf{i}, \mathbf{j}}^{(1)},
$$

which implies that $\left(x_{1}, \ldots, x_{n}\right)$ is $E_{1}(n)$-invariant.

Given an orthogonal Hopf algebra $E(n)$ generated by $\left\{u_{i, j}\right\}_{i, j=1, \ldots, n}$. Then, for $k \in \mathbb{N}, E(n)$ can be considered as an orthogonal Hopf algebra $E(n, k)$ generated by $\left\{v_{i, j}\right\}_{i, j=1, \ldots, n+k}$ such that

$$
v_{i, j}=\left\{\begin{array}{ll}
u_{i, j} & \text { if } i, j \leq n \\
\delta_{i, j} 1_{E(n)} & \text { otherwise }
\end{array} .\right.
$$


We will call $E(n, k)$ the $k$-th extension of $E(n)$. To study de Finetti type theorems for all orthogonal Hopf algebras $E(n)$, we need to extend $E(n)$-invariance condition on $n$ random variables to an invariance condition on infinitely many random variables.

Definition 3.11. Given a probability space $(\mathcal{A}, \phi)$, a sequence of random variables $\left(x_{i}\right)_{i \in \mathbb{N}}$ of $\mathcal{A}$ and an orthogonal Hopf algebra $E(n)$ generated by $\left\{u_{i, j}\right\}_{i, j=1, \ldots n}$. We say that the joint distribution $\mu$ of $\left(x_{i}\right)_{i \in \mathbb{N}}$ is $E(n)$-invariant if the joint distribution of $\left(x_{1}, \ldots, x_{n+k}\right)$ is $E(n, k)$ invariant for all $k \in \mathbb{N}$.

\section{Boolean Quantum Semigroups in analogous to EASy quantum Groups}

Inspired by the previous work in [12], we will define distributional symmetries for Boolean independent random variables via quantum semigroups. For any $C^{*}$-algebras $A$ and $B$, the set of morphisms $\operatorname{Mor}(A, B)$ consists of all $C^{*}$-algebra homomorphisms acting from $A$ to $M(B)$, where $M(B)$ is the multiplier algebra of $B$, such that $\phi(A) B$ is dense in $B$. If $A$ and $B$ are unital $C^{*}$-algebras, then all unital $C^{*}$-homomorphisms from $A$ to $B$ are in Mor(A,B). In [17], the morphisms in the category of quantum semigroups are defined as follows.

Definition 4.1. By a quantum semigroup we mean a $C^{*}$-algebra $\mathcal{A}$ endowed with an additional structure described by a morphism $\Delta \in \operatorname{Mor}(\mathcal{A}, \mathcal{A} \otimes \mathcal{A})$ such that

$$
\left(\Delta \otimes i d_{\mathcal{A}}\right) \Delta=\left(i d_{\mathcal{A}} \otimes \Delta\right) \Delta .
$$

The quantum semigroups for Boolean independence are unital universal $C^{*}$-algebras generated by an orthogonal projection $\mathbf{P}$ and entries of $n \times n$ matrices $u=\left(u_{i, j}\right)_{i, j=1, \ldots, n}$ which satisfy certain relation $R$ related to $\mathbf{P}$.

Definition 4.2. Let $u=\left(u_{i, j}\right)_{i, j=1, \ldots, n} \in M_{n}(\mathcal{A})$ be an $n \times n$ matrix over a $C^{*}$-algebra $\mathcal{A}$ and $\mathbf{P}$ be an orthogonal projection in $\mathcal{A}$. The pair $(u, \mathbf{P})$ is said to be:

(1) $\mathbf{P}$-orthogonal, if all entries of $u$ are selfadjoint, and $u u^{t}\left(1_{n} \otimes \mathbf{P}\right)=u^{t} u\left(1_{n} \otimes \mathbf{P}\right)=1_{n} \otimes \mathbf{P}$ i.e. $\sum_{k=1}^{n} u_{i, k} u_{j, k} \mathbf{P}=\sum_{k=1}^{n} u_{k, i} u_{k, j} \mathbf{P}=\delta_{i, j} \mathbf{P}$.

(2) $\mathbf{P}$-magic, if it is $\mathbf{P}$-orthogonal and the entries of $u$ are projections.

(3) $\mathbf{P}$-cubic, if it is $\mathbf{P}$-orthogonal and $u_{i, j} u_{i, k} \mathbf{P}=u_{j, i} u_{j, k} \mathbf{P}=0$, for $j \neq k$.

(4) $\mathbf{P}$-bistochastic, if it is $\mathbf{P}$-orthogonal, and $\sum_{j=1}^{n} u_{i, j} \mathbf{P}=\sum_{k=1}^{n} u_{k, i} \mathbf{P}=\mathbf{P}$, for all $i$.

(5) $\mathbf{P}_{-} '$, if $\sum_{j=1}^{n} u_{i, j} \mathbf{P}=\sum_{k=1}^{n} u_{k, i} \mathbf{P}$, for all $i$.

(6) $\mathbf{P}$-magic', if it is $\mathbf{P}$-cubic and $\mathbf{P}$-'.

(7) $\mathbf{P}$-bistochastic', if it is $\mathbf{P}$-orthogonal and $\mathbf{P}$-'.

Unlike universal conditions for quantum groups, these conditions cannot define universal $C^{*}$-algebras since they cannot ensure that $u_{i, j}$ 's are bounded. Therefore, we need an additional condition to control the norms of $u_{i, j}^{\prime} \mathrm{s}$. We say $\left(u_{i, j}\right)_{i,=1, \ldots n}$ is norm $\leq 1$ if the norm $\left\|\left(u_{i, j}\right)_{i, j=1, \ldots, n}\right\| \leq 1$.

Definition 4.3. Let $B_{g}(n)$ with $g=o, s, h, b, s^{\prime}, b^{\prime}$ be the unital universal $C^{*}$-algebra generated by the entries of a norm $\leq 1$ matrix $u=\left(u_{i, j}\right)_{i,=1, \ldots n}$ and an orthogonal projection $\mathbf{P}$ when the pair $(u, \mathbf{P})$ is $\mathbf{P}$-orthogonal, $\mathbf{P}$-magic, $\mathbf{P}$-cubic, $\mathbf{P}$-bistochastic, $\mathbf{P}$-magic' and $\mathbf{P}$-bistochastic', respectively. 
On the $C^{*}$-algebra $B_{g}(n)$ with $g=o, s, h, b, s^{\prime}, b^{\prime}$, we can always define a unital $C^{*}$-homomorphism

$$
\Delta: B_{g}(n) \rightarrow B_{g}(n) \otimes B_{g}(n)
$$

on their generators by the following equations:

$$
\Delta u_{i, j}=\sum_{k=1}^{n} u_{i, k} \otimes u_{k, j}
$$

and

$$
\Delta \mathbf{P}=\mathbf{P} \otimes \mathbf{P}, \quad \Delta I=I \otimes I .
$$

To show the coproduct $\Delta$ is well defined, we need to show that the $\left(\Delta u_{i, j}\right)_{i, j=1, \ldots, n}$ and $\mathbf{P} \otimes \mathbf{P}$ satisfy the universal conditions as $\left(u_{i, j}\right)_{i, j=1, \ldots, n}$ and $\mathbf{P}$ do. We check them below.

Norm condition: If $\left\|\left(u_{i, j}\right)_{i, j=1, \ldots n}\right\| \leq 1$, then we have

$\left\|\left(\Delta u_{i, j}\right)_{i, j=1, \ldots n}\right\|=\left\|\left(\sum_{k=1}^{n} u_{i, k} \otimes u_{k, j}\right)_{i, j=1, \ldots n}\right\|=\left\|\left(u_{i, j} \otimes 1_{n}\right)_{i, j=1, \ldots n}\left(1_{n} \otimes u_{i, j}\right)_{i, j=1, \ldots n}\right\| \leq\left\|\left(u_{i, j}\right)_{i, j=1, \ldots n}\right\|^{2} \leq 1$.

P-orthogonal: If $\sum_{k=1}^{n} u_{i, k} u_{j, k} \mathbf{P}=\sum_{k=1}^{n} u_{k, i} u_{k, j} \mathbf{P}=\delta_{i, j} \mathbf{P}$, then

$$
\begin{aligned}
\sum_{k=1}^{n} \Delta u_{i, k} \Delta u_{j, k} \Delta \mathbf{P} & =\sum_{k=1}^{n}\left(\sum_{l=1}^{n} u_{i, l} \otimes u_{l, k}\right)\left(\sum_{m=1}^{n} u_{j, m} \otimes u_{m, k}\right)(\mathbf{P} \otimes \mathbf{P}) \\
& =\sum_{k=1}^{n} \sum_{l=1}^{n} \sum_{m=1}^{n} u_{i, l} u_{j, m} \mathbf{P} \otimes u_{l, k} u_{m, k} \mathbf{P} \\
& =\sum_{l=1}^{n} \sum_{m=1}^{n} u_{i, l} u_{j, m} \mathbf{P} \otimes \delta_{m, l} \mathbf{P} \\
& =\sum_{l=1}^{n} u_{i, l} u_{j, l} \mathbf{P} \otimes \mathbf{P} \\
& =\delta_{i, j} \mathbf{P} \otimes \mathbf{P} .
\end{aligned}
$$

Similarly, we have $\sum_{k=1}^{n} \Delta u_{k, i} \Delta u_{k, j} \Delta \mathbf{P}=\delta_{i, j} \mathbf{P} \otimes \mathbf{P}$.

P-cubic: Assume that $u_{i, j} u_{i, k} \mathbf{P}=u_{j, i} u_{j, k} \mathbf{P}=0$, for $j \neq k$, then we have

$$
\begin{aligned}
\Delta u_{i, j} \Delta u_{i, k} \Delta \mathbf{P} & =\sum_{l, m=1}^{n} u_{i, l} u_{i, m} \mathbf{P} \otimes u_{l, j} u_{m, k} \mathbf{P} \\
& =\sum_{l=1}^{n} u_{i, l} u_{i, l} \mathbf{P} \otimes u_{l, j} u_{l, k} \mathbf{P} \\
& =0
\end{aligned}
$$

whenever $j \neq k$. Similarly, we have

$$
\Delta u_{j, i} \Delta u_{j, k} \Delta \mathbf{P}=0
$$


whenever $j \neq k$.

P-bistochastic: If $\sum_{j=1}^{n} u_{i, j} \mathbf{P}=\sum_{j=1}^{n} u_{j, i} \mathbf{P}=\mathbf{P}$, for all $j=1, \ldots, n$. Then we have

$$
\sum_{j=1}^{n} \Delta u_{i, j} \Delta \mathbf{P}=\sum_{j=1}^{n} \sum_{k=1}^{n} u_{i, k} \mathbf{P} \otimes u_{k, j} \mathbf{P}=\sum_{j=1}^{n} u_{i, j} \mathbf{P} \otimes \mathbf{P}=\mathbf{P} \otimes \mathbf{P} .
$$

Similarly, we have $\sum_{j=1}^{n} \Delta u_{j, i} \Delta \mathbf{P}=\mathbf{P} \otimes \mathbf{P}$, for all $j$.

$\mathbf{P}^{\prime}$-condition: Let $r=\sum_{j=1}^{n} u_{i, j} \mathbf{P}=\sum_{j=1}^{n} u_{j, i} \mathbf{P}$, for $j \neq k$. Then we have

$$
\sum_{j=1}^{n} \Delta u_{i, j} \Delta \mathbf{P}=\sum_{j, l=1}^{n} u_{i, l} \mathbf{P} \otimes u_{l, j} \mathbf{P}=\sum_{l=1}^{n} u_{i, l} \mathbf{P} \otimes r=r \otimes r
$$

for all $j$. Similarly, we have $\sum_{j=1}^{n} \Delta u_{j, i} \Delta \mathbf{P}=r \otimes r$ for all $j$.

Therefore, $\Delta$ is a well defined $C^{*}$-homomorphism and $\left(B_{g}(n), \Delta\right)$ is a quantum semigroup for $g=o, s, h, b, s^{\prime}, b^{\prime}$. As the relations for easy quantum groups, we have the following diagram for Boolean quantum semigroups:

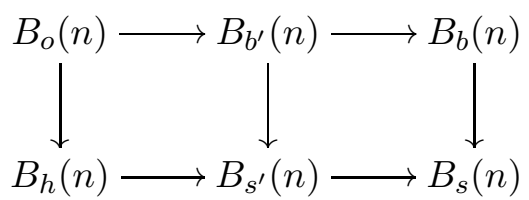

Furthermore, for $g=o, s, h, b, s^{\prime}, b^{\prime}, A_{g}(n)$ is a quotient algebra of $B_{g}(n)$ by requiring $\mathbf{P}$ to be the unit of the algebra. Therefore, we have

$$
C_{g}(n) \subseteq A_{g}(n) \subseteq B_{g}(n)
$$

for $g=o, s, h, b, s^{\prime}, b^{\prime}$.

In addition, the algebras $B_{g}(n)$ generated by the generators of $B_{g}(n)$ with $g=o, s, h, b$ are quotient algebras of Hayase's Hopf algebras $C\left(G_{n}^{I_{2}}\right), C\left(G_{n}^{I}\right), C\left(G_{n}^{I_{h}}\right), C\left(G_{n}^{I_{b}}\right)$ in [7], respectively. Indeed, $B_{g}(n)$ with $g=o, s, h, b$ satisfy Hayase's universal conditions for $C\left(G_{n}^{I_{2}}\right), C\left(G_{n}^{I}\right), C\left(G_{n}^{I_{h}}\right), C\left(G_{n}^{I_{b}}\right)$, respectively. To check the vanishing conditions, we apply the following notation for convenience: Given $\pi_{1} \in I\left(k_{1}\right)$ and $\pi_{2} \in I\left(k_{2}\right), \pi=\pi_{1} \pi_{2} \in I\left(k_{1}+k_{2}\right)$ denotes the concatenation of $\pi_{1}$ and $\pi_{2}$. Given $\mathbf{j}_{1}=\left(j_{1}, \ldots, j_{k_{1}}\right) \in[n]^{k_{1}}$ and $\mathbf{j}_{2}=\left(j_{1}^{\prime}, \ldots, j_{k_{2}}^{\prime}\right) \in[n]^{k_{2}}, \mathbf{j}=\mathbf{j}_{1} \mathbf{j}_{2}=\left(j_{1}, \ldots, j_{k_{1}}, j_{1}^{\prime}, \ldots, j_{k_{2}}^{\prime}\right) \in$ $[n]^{k_{1}+k_{2}}$. According to Definition $[2.9$, the following lemma is obvious.

Lemma 4.4. Let $\pi \in I\left(k_{1}+k_{2}\right)$ such that $\pi=\pi_{1} \pi_{2}$ for some $\pi_{1} \in I\left(k_{1}\right)$ and $\pi_{2} \in P\left(k_{2}\right)$. Let $\mathbf{j}=\mathbf{j}_{1}+\mathbf{j}_{2}$ such that $\mathbf{j}_{1} \in[n]^{k_{1}}$ and $\mathbf{j}_{2} \in[n]^{k_{2}}$. Then, $\pi \leq \operatorname{ker} \mathbf{j}$ iff $\pi_{i} \leq \operatorname{ker} \mathbf{j}_{i}$ for $i=1,2$.

Therefore, we have the following property.

Lemma 4.5. Given $\pi_{1} \in I\left(k_{1}\right), \pi_{2} \in P\left(k_{2}\right)$ and $\mathbf{j}=\mathbf{j}_{1}+\mathbf{j}_{2}$ such that $\mathbf{j}_{1} \in[n]^{k_{1}}$ and $\mathbf{j}_{2} \in[n]^{k_{2}}$. If

$$
\sum_{\mathbf{i}_{i} \in[n]^{k_{i}, \pi_{i} \leq \operatorname{ker} \mathbf{i}_{i}}} u_{\mathbf{i}_{i}, \mathbf{j}_{i}} \mathbf{P}= \begin{cases}\mathbf{P} & \text { if } \pi \leq \operatorname{ker} \mathbf{j}_{i} \\ 0 & \text { otherwise }\end{cases}
$$


for $i=1,2$. Then, we have

$$
\sum_{\mathbf{i} \in[n]^{k_{1}+k_{2}, \pi_{1} \pi_{2} \leq \text { ker } \mathbf{i}}} u_{\mathbf{i}, \mathbf{j}} \mathbf{P}= \begin{cases}\mathbf{P} & \text { if } \pi \leq \text { ker } \mathbf{j} \\ 0 & \text { otherwise }\end{cases}
$$

Proof. By a direct computation, we have:

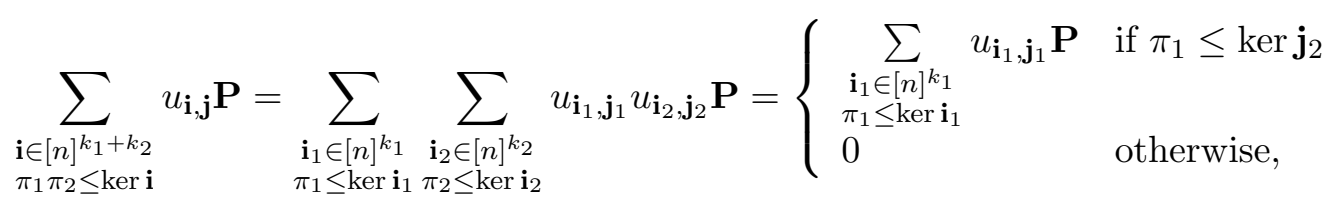

Therefore,

$$
\sum_{\substack{\mathbf{i} \in[n]^{k_{1}+k_{2}} \\
\pi_{1} \pi_{2} \leq \text { ker } \mathbf{i}}} u_{\mathbf{i}, \mathbf{j}} \mathbf{P}=\left\{\begin{array}{cc}
\mathbf{P} & \pi_{1} \leq \operatorname{ker} \mathbf{j}_{1} \text { and } \pi_{2} \leq \operatorname{ker} \mathbf{j}_{2} \\
0 & \text { otherwise }
\end{array}\right.
$$

which completes the proof.

Now, we turn to check the following vanishing conditions.

Lemma 4.6. Let $u_{i, j}$ 's and $\mathbf{P}$ be the standard generators of $B_{o}(n), B_{s}(n), B_{h}(n), B_{b}(n)$. Then, we have

$$
\sum_{\mathbf{i} \in[n]^{k}, \pi \leq \text { ker } \mathbf{i}} u_{\mathbf{i}, \mathbf{j}} \mathbf{P}= \begin{cases}\mathbf{P} & \text { if } \pi \leq \mathrm{ker} \mathbf{j} \\ 0 & \text { otherwise }\end{cases}
$$

for $\pi \in I_{2}(k), I(k), I_{h}(k), I_{b}(k)$, respectively.

Proof. 1. For $B_{o}(n), k=2$. The identity holds by the definition of $B_{o}(n)$. Since all partitions in $I_{2}(n)$ are concatenations of pair partitions by Lemma 4.5, the equation holds.

2. For $B_{b}(n)$, the identity holds by the definition of $B_{b}(n)$ when $\pi$ is a single partition or a partition. Since all partitions in $I_{b}(n)$ are concatenations of single partitions and pair partitions, by Lemma 4.5, the equation holds.

3. For $B_{h}(n)$ we need to check $\pi=1_{2 m} \in I_{h}(2 m)$ for all $m \in \mathbb{N}$. It follows that

$$
\sum_{\substack{\mathbf{i} \in[n]^{2 m} \\ \pi \leq \operatorname{ker} \mathbf{i}}} u_{\mathbf{i}, \mathbf{j}} \mathbf{P}=\sum_{i=1}^{n} u_{i, j_{1}} \cdots u_{i, j_{2 m}} \mathbf{P}
$$

It equals zero if $j_{l} \neq j_{l+1}$ for some $l$, otherwise

$$
\sum_{i=1}^{n} u_{i, j_{1}} \cdots u_{i, j_{2 m}} \mathbf{P}=\sum_{i=1}^{n} u_{i, j_{1}}^{2 m} \mathbf{P}=\sum_{i=1}^{n} u_{i, j_{1}}^{2 m-2} \sum_{l=1}^{n} u_{l, j_{1}}^{2} \mathbf{P}=\sum_{i=1}^{n} u_{i, j_{1}}^{2 m-2} \mathbf{P}=\cdots=\sum_{l=1}^{n} u_{l, j_{1}}^{2} \mathbf{P}=\mathbf{P} .
$$

Since all partitions in $I_{b}(n)$ are concatenations of blocks of even length, by Lemma 4.5, the equation holds.

4. For $B_{s}(n)$, we need to check $\pi=1_{m} \in I(m)$, for all $m \in \mathbb{N}$. It follows that

$$
\sum_{\substack{\mathbf{i} \in[n]^{m} \\ \pi \leq \mathrm{ker} \mathbf{i}}} u_{\mathbf{i}, \mathbf{j}} \mathbf{P}=\sum_{i=1}^{n} u_{i, j_{1}} \cdots u_{i, j_{m}} \mathbf{P} .
$$

It equals zero if $j_{l} \neq j_{l+1}$ for some $l$, otherwise

$$
\sum_{i=1}^{n} u_{i, j_{1}} \cdots u_{i, j_{m}} \mathbf{P}=\sum_{i=1}^{n} u_{i, j_{1}}^{m} \mathbf{P}=\sum_{i=1}^{n} u_{i, j_{1}}^{m-1} \sum_{l=1}^{n} u_{l, j_{1}} \mathbf{P}=\sum_{i=1}^{n} u_{i, j_{1}}^{m-1} \mathbf{P}=\cdots=\sum_{l=1}^{n} u_{l, j_{1}} \mathbf{P}=\mathbf{P} .
$$


Since all partitions in $I_{b}(n)$ are concatenations of blocks of arbitrary length, by Lemma 4.5, the equation holds.

The noncommutative distributional symmetries for Boolean independence are defined as follows.

Definition 4.7. An orthogonal Boolean quantum semigroup is a unital $C^{*}$-algebra $A$ generated by $n^{2}$ selfadjoint elements $\left\{u_{i . j} \mid i, j=1, \ldots, n\right\}$ and an orthogonal projection $\mathbf{P}$, such that the following hold:

1. $u=\left(u_{i, j}\right)_{i, j=1, \ldots . n} \in M_{n}(A)$ such that $\|u\| \leq 1$ and $(\mathrm{u}, \mathbf{P})$ is $\mathbf{P}$-orthogonal.

2. $\Delta\left(u_{i, j}\right)=\sum_{k=1}^{n} u_{i, k} \otimes u_{k, j}$ and $\Delta \mathbf{P}=\mathbf{P} \otimes \mathbf{P}, \Delta I=I \otimes I$ determine a $C^{*}$-unital homomorphism $\Delta: A \rightarrow A \otimes_{\min } A$.

Definition 4.8. Let $(A, \Delta)$ be a quantum semigroup and $\mathcal{V}$ be a unital algebra. By a right coaction of the quantum semigroup $A$ on $\mathcal{V}$, we mean a unital homomorphism $\alpha: \mathcal{V} \rightarrow \mathcal{V} \otimes \mathcal{A}$ such that

$$
\left(\alpha \otimes i d_{A}\right) \alpha=(i d \otimes \Delta) \alpha .
$$

Definition 4.9. Given an orthogonal Boolean quantum semigroup $E(n)$ generated by $\left\{u_{i, j}\right\}_{i, j=1, \ldots n}$ and $\mathbf{P}$, we have a natural coaction $\alpha_{n}$ of $E(n)$ on $\mathbb{C}\left\langle X_{1}, \ldots, X_{n}\right\rangle$ such that

$$
\alpha_{n}: \mathbb{C}\left\langle X_{1}, \ldots, X_{n}\right\rangle \rightarrow \mathbb{C}\left\langle X_{1}, \ldots, X_{n}\right\rangle \otimes E(n)
$$

is a homomorphism defined via $\alpha_{n}\left(X_{i}\right)=\sum_{k=1}^{n} X_{k} \otimes u_{k, i}$ for all $i$.

Definition 4.10. Given a probability space $(\mathcal{A}, \phi)$, a sequence of random variables $\left(x_{1}, \ldots, x_{n}\right)$ of $\mathcal{A}$ and an orthogonal Boolean quantum semigroup $E(n)$ generated by $\left\{u_{i, j}\right\}_{i, j=1, \ldots n}$ and $\mathbf{P}$. We say that the joint distribution $\mu_{x_{1}, \ldots, x_{n}}$ of $x_{1}, \ldots, x_{n}$ is $E(n)$-invariant if

$$
\mu_{x_{1}, \ldots, x_{n}}(p) \mathbf{P}=\mu_{x_{1}, \ldots, x_{n}} \otimes i d_{E(n)}\left(\alpha_{n}(p)\right) \mathbf{P},
$$

for all $p \in \mathbb{C}\left\langle X_{1}, \ldots, X_{n}\right\rangle$.

As orthogonal Hopf algebras, we can define $E(n)$-invariance condition for an infinite sequence of random variables. Given an orthogonal Boolean quantum semigroup $E(n)$ generated by $\left\{u_{i, j}\right\}_{i, j=1, \ldots, n}$ and $\mathbf{P}$, then for $k \in \mathbb{N}, E(n)$ can be considered as an orthogonal Boolean quantum semigroup $E(n, k)$ generated by $\left\{v_{i, j}\right\}_{i, j=1, \ldots, n+k}$ and $\mathbf{P}^{\prime}$ such that

$$
v_{i, j}= \begin{cases}u_{i, j} & \text { if } i, j \leq n \\ \delta_{i, j} 1_{E(n)} & \text { otherwise }\end{cases}
$$

and $\mathbf{P}^{\prime}=\mathbf{P}$. We will call $E(n, k)$ the $k$-th extension of $E(n)$.

Definition 4.11. Given a probability space $(\mathcal{A}, \phi)$, a sequence of random variables $\left(x_{i}\right)_{i \in \mathbb{N}}$ and an orthogonal Hopf algebra $E(n)$ generated by $\left\{u_{i, j}\right\}_{i, j=1, \ldots n}$. We say that the joint distribution $\mu$ of $\left(x_{i}\right)_{i \in \mathbb{N}}$ is $E(n)$-invariant if the joint distribution of $\left(x_{1}, \ldots, x_{n+k}\right)$ is $E(n, k)$-invariant for all $k \in \mathbb{N}$.

Proposition 4.12. Let $(\mathcal{A}, \mathcal{B}, \mathbb{E}: \mathcal{A} \rightarrow \mathcal{B})$ be an operator valued probability space and $\left\{x_{i}\right\}_{i=1, \ldots, n}$ be a sequence of random variables in $\mathcal{A}$. Let $\phi$ be a linear functional on $\mathcal{A}$ such that $\phi(\cdot)=\phi(\mathbb{E}[\cdot])$. Then, we have

- If $\left(x_{i}\right)_{i=1, \ldots, n}$ is identically distributed and Boolean independent with respect to $\mathbb{E}$, then the sequence is $B_{s}(n)$-invariant.

- If $\left(x_{i}\right)_{i=1, \ldots, n}$ is identically symmetric distributed and Boolean independent with respect to $\mathbb{E}$, then the sequence is $B_{h}(n)$-invariant. 
- If $\left(x_{i}\right)_{i=1, \ldots, n}$ has identically shifted Bernoulli distribution and is Boolean independent with respect to $\mathbb{E}$, then the sequence is $B_{b}(n)$-invariant.

- If $\left(x_{i}\right)_{i=1, \ldots, n}$ has identically centered Bernoulli distribution and Boolean independent with respect to $\mathbb{E}$, then the sequence is $B_{o}(n)$-invariant.

Proof. Suppose that the joint distribution of $\left\{x_{i}\right\}_{i=1, \ldots, n}$ satisfies one of the conditions specified in the statement of the proposition, and let $D(k)$ be the partition family associated to the corresponding quantum semigroups. Let $X_{\mathbf{j}}=X_{j_{1}} \cdots X_{j_{k}}$, by Lemma 4.6 and 2.14, we have

$$
\begin{aligned}
& \mu_{x_{1}, \ldots, x_{n}}\left(\alpha_{n}\left(X_{\mathbf{j}}\right)\right) \mathbf{P}=\sum_{\mathbf{i} \in[n]^{k}} \mu_{x_{1}, \ldots, x_{n}}\left(X_{\mathbf{i}}\right) u_{\mathbf{i}, \mathbf{j}} \mathbf{P} \\
& =\sum_{\mathbf{i} \in[n]^{k}} \phi\left(x_{\mathbf{i}}\right) u_{\mathbf{i}, \mathbf{j}} \mathbf{P} \\
& =\sum_{\mathbf{i} \in[n]^{k}} \phi\left(\mathbb{E}\left[x_{\mathbf{i}}\right]\right) u_{\mathbf{i}, \mathbf{j}} \mathbf{P} \\
& =\sum_{\mathbf{i} \in[n]^{k}} \sum_{\pi \in D(k)} \phi\left(b_{E}^{(\pi)}\left(x_{\mathbf{i}}\right)\right) u_{\mathbf{i}, \mathbf{j}} \mathbf{P} \\
& =\sum_{\pi \in D(k)} \sum_{\mathbf{i} \in[n]^{k}} \phi\left(b_{E}^{(\pi)}\left(x_{\mathbf{i}}\right)\right) u_{\mathbf{i}, \mathbf{j}} \mathbf{P} \\
& =\sum_{\pi \in D(k)} \sum_{\substack{\mathbf{i} \in[n]^{k} \\
\pi \leq \mathrm{ker} \mathbf{i}}} \phi\left(b_{E}^{(\pi)}\left(x_{\mathbf{i}}\right)\right) u_{\mathbf{i}, \mathbf{j}} \mathbf{P} \\
& =\sum_{\pi \in D(k)} \sum_{\substack{\mathbf{i} \in[n]^{k} \\
\pi \leq \mathrm{ker} \mathbf{i}}} \phi\left(b_{E}^{(\pi)}\left(x_{1}, \ldots, x_{1}\right)\right) u_{\mathbf{i}, \mathbf{j}} \mathbf{P} \\
& =\sum_{\substack{\pi \in D(k) \\
\pi \leq \operatorname{ker} \mathbf{j}}} \phi\left(b_{E}^{(\pi)}\left(x_{1}, \ldots, x_{1}\right)\right) \mathbf{P} \\
& =\sum_{\substack{\pi \in D(k) \\
\pi \leq \operatorname{ker} \mathbf{j}}} \phi\left(b_{E}^{(\pi)}\left(x_{\mathbf{j}}\right)\right) \mathbf{P} \\
& =\phi\left(\mathbb{E}\left[x_{\mathbf{j}}\right]\right) \mathbf{P} \\
& =\phi\left(x_{\mathbf{j}}\right) p \\
& =\mu_{x_{1}, \ldots, x_{n}}\left(X_{\mathbf{j}}\right) \mathbf{P},
\end{aligned}
$$

which completes the proof.

\section{MAin RESUlt}

In this section, we will prove Theorem 1.1 and Theorem 1.2. Then, we present an application of these theorems to easy groups $C_{s^{\prime}}(n)$ and $C_{b^{\prime}}(n)$, easy quantum groups $A_{s^{\prime}}(n), A_{b^{\prime}}(n)$ and $A_{b^{\#}}(n)$, Boolean quantum semigroups $B_{s^{\prime}}(n)$ and $B_{b^{\prime}}(n)$. We start with free independence, since the proof of the other parts can be easily derived from this case.

Given a $W^{*}$-probability space $(\mathcal{A}, \phi)$ such that $\phi$ is faithful. Let $\{E(n)\}_{n \in \mathbb{N}}$ be a sequence of orthogonal Hopf algebras such that $A_{s}(n) \subseteq E(n) \subseteq A_{o}(n)$ for each $n$. Let $\left(x_{i}\right)_{i \in \mathbb{N}}$ be a sequence of random variables which generate $A$. Suppose that the joint distribution of $\left(x_{i}\right)_{i \in \mathbb{N}}$ is 
$E(n)$-invariant for all $n$. By Proposition $3.10,\left(x_{i}\right)_{i \in \mathbb{N}}$ are $A_{s}(n)$-invariant for all $n$. By Köstler and Speicher [10], there exist a $W^{*}$-subalgebra $\mathcal{B}$ such that $1 \subseteq \mathcal{B} \subseteq \mathcal{A}$ and a $\phi$-preserving conditional expectation $\mathbb{E}: \mathcal{A} \rightarrow \mathcal{B}$ such that $\left(x_{i}\right)_{i \in \mathbb{N}}$ are freely independent and identically distributed with respect to $\mathbb{E}$. This is exactly the statement 1 for the free case. In addition, by Proposition 4.3 in [10] and Definition [3.11, the coaction invariant condition for $\phi$ can be extended to the conditional expectation $\mathbb{E}$, i.e.

$$
\mathbb{E}\left[b_{0} x_{i_{1}} b_{1} \cdots b_{k-1} x_{i_{k}} b_{k}\right] \otimes 1_{E(n)}=\sum_{j_{1}, \ldots, j_{k}=1}^{n} \mathbb{E}\left[b_{0} x_{j_{1}} b_{1} \cdots b_{k-1} x_{j_{k}} b_{k}\right] \otimes u_{j_{1}, i_{1}} \cdots u_{j_{k}, i_{k}}
$$

for $i_{1}, \ldots, i_{k} \leq n$, where $\left\{u_{i, j} \mid i, j=1, \ldots, n\right\}$ are generators of $E(n)$.

2. Suppose that $A_{s}(n) \subseteq E(n) \subseteq A_{b}(n)$ for all $n$ and there exists a number $k$ such that $E(k) \neq A_{s}(k)$. Let $\left\{u_{i, j}\right\}_{i, j=1, \ldots, k}$ be generators of $E(k)$. By proposition 3.5, there exists an index $i^{\prime}$ such that

$$
\sum_{l=1}^{k} u_{l, i^{\prime}}^{m} \neq 1
$$

for all $m>2$. Without loss of generality, we assume that $i^{\prime}=1$. It is sufficient to show that $\kappa_{l}\left(x_{1} b_{1}, \ldots, x_{1} b_{l}\right)=0$ for all $l \geq 3$, where $b_{1}, \ldots, b_{l} \in B$. We check it by induction on $l$. First, we have that

$$
\begin{aligned}
& \mathbb{E}\left[x_{1} b_{1} \cdots x_{1} b_{l}\right] \otimes 1_{E(n)} \\
& =\sum_{\mathbf{i} \in[k]^{l}} \mathbb{E}\left[x_{i_{1}} b_{1} \cdots x_{i_{l}} b_{l}\right] \otimes u_{\mathbf{i}, 1} \\
& =\sum_{\mathbf{i} \in[k]^{l}} \sum_{\pi \in N C(l)} \kappa_{\pi}\left(x_{i_{1}} b_{1}, \ldots, x_{i_{l}} b_{l}\right) \otimes u_{\mathbf{i}, 1} \\
& =\sum_{\pi \in N C_{b}(l)} \sum_{\mathbf{i} \in[k]^{l}} \kappa_{\pi}\left(x_{i_{1}} b_{1}, \ldots, x_{i_{l}} b_{l}\right) \otimes u_{\mathbf{i}, 1}+\sum_{\pi \in N C(l) \backslash N C_{b}(l)} \sum_{\mathbf{i} \in[k]^{l}} \kappa_{\pi}\left(x_{i_{1}} b_{1}, \ldots, x_{i_{l}} b_{l}\right) \otimes u_{\mathbf{i}, 1} \\
& =\sum_{\pi \in N C_{b}(l)} \sum_{\substack{\mathbf{i} \in[k]^{l} \\
\pi \leq \mathrm{ker} \mathbf{i}}} \kappa_{\pi}\left(x_{i_{1}} b_{1}, \ldots, x_{i_{l}} b_{l}\right) \otimes u_{\mathbf{i}, 1}+\sum_{\pi \in N C(l) \backslash N C_{b}(l)} \sum_{\substack{\mathbf{i} \in[k]^{l} \\
\pi \leq \operatorname{ker} \mathbf{i}}} \kappa_{\pi}\left(x_{i_{1}} b_{1}, \ldots, x_{i_{l}} b_{l}\right) \otimes u_{\mathbf{i}, 1} \\
& =\sum_{\pi \in N C_{b}(l)} \sum_{\substack{\mathbf{i} \in[k]^{l} \\
\pi \leq \operatorname{ker} \mathbf{i}}} \kappa_{\pi}\left(x_{1} b_{1}, \ldots, x_{1} b_{l}\right) \otimes u_{\mathbf{i}, 1}+\sum_{\pi \in N C(l) \backslash N C_{b}(l)} \sum_{\substack{\mathbf{i} \in[k]^{l} \\
\pi \leq \operatorname{ker} \mathbf{i}}} \kappa_{\pi}\left(x_{1} b_{1}, \ldots, x_{1} b_{l}\right) \otimes u_{\mathbf{i}, 1} \\
& =\sum_{\pi \in N C_{b}(l)} \kappa_{\pi}\left(x_{1} b_{1}, \ldots, x_{1} b_{l}\right) \otimes 1_{E(n)}+\sum_{\pi \in N C(l) \backslash N C_{b}(l)} \sum_{\substack{\mathbf{i} \in[k]^{l} \\
\pi \leq \operatorname{ker} \mathbf{i}}} \kappa_{\pi}\left(x_{1} b_{1}, \ldots, x_{1} b_{l}\right) \otimes u_{\mathbf{i}, 1} .
\end{aligned}
$$

The first term of the last equality follows because $E(n)$ is a quotient algebra of $A_{b}(n)$. On the other hand, we have that

$\mathbb{E}\left[x_{1} b_{1}, \ldots, x_{1} b_{l}\right] \otimes 1_{E(n)}=\sum_{\pi \in N C_{b}(k)} \kappa_{\pi}\left(x_{1} b_{1}, \ldots, x_{1} b_{l}\right) \otimes 1_{E(n)}+\sum_{\pi \in N C(l) \backslash N C_{b}(l)} \kappa_{\pi}\left(x_{1} b_{1}, \ldots, x_{1} b_{l}\right) \otimes 1_{E(n)}$.

It follows that

$$
\sum_{\pi \in N C(l) \backslash N C_{b}(l)} \sum_{\substack{\mathbf{i} \in[k]^{l} \\ \pi \leq \operatorname{ker} \mathbf{i}}} \kappa_{\pi}\left(x_{1} b_{1}, \ldots, x_{1} b_{l}\right) \otimes u_{\mathbf{i}, 1}=\sum_{\pi \in N C(l) \backslash N C_{b}(l)} \kappa_{\pi}\left(x_{1} b_{1}, \ldots, x_{1} b_{l}\right) \otimes 1_{E(n)} .
$$


When $l=3$, we have $N C(3) \backslash N C_{b}(3)=\left\{1_{3}\right\}$. Then

$$
\sum_{\substack{\mathbf{i} \in[n]^{k} \\ \pi \leq \operatorname{ker} 1_{3}}} \kappa_{1_{3}}\left(x_{1} b_{1}, \ldots, x_{1} b_{3}\right) \otimes u_{\mathbf{i}, 1}=\kappa_{1_{3}}\left(x_{1} b_{1}, \ldots, x_{1} b_{3}\right) \otimes 1_{E(n)},
$$

which is

$$
\kappa_{1_{3}}\left(x_{1} b_{1}, \ldots, x_{1} b_{k}\right) \otimes\left(\sum_{l=1}^{k} u_{l, 1}^{3}-1_{E(n)}\right)=0 .
$$

Therefore, $\kappa_{1_{3}}\left(x_{1} b_{1}, \ldots, x_{1} b_{3}\right)=0$. Suppose $\kappa_{1_{l}}\left(x_{1} b_{1}, \ldots, x_{1} b_{l}\right)=0$ for $3 \leq l \leq m$, then for $\pi \in N C(m+1), \kappa_{\pi}\left(x_{i_{1}} b_{1}, \ldots, x_{1} b_{m+1}\right)=0$ if $\pi$ contains a block whose size is between 3 and $m$. Each partition $\pi \in N C(m+1) \backslash N C_{b}(m+1)$ contains at least one block whose size is greater than 2. Therefore, for $\pi \in N C(m+1) \backslash N C_{b}(m+1), \kappa_{\pi}\left(x_{1} b_{1}, \ldots, x_{1} b_{k}\right)=0$ if $\pi \neq 1_{m+1}$. Hence, equation 1 becomes

$$
\kappa_{1_{m+1}}\left(x_{1} b_{1}, \ldots, x_{1} b_{m+1}\right) \otimes\left(\sum_{l=1}^{k} u_{l, 1}^{m+1}-1_{E(n)}\right)=0
$$

which implies

$$
\kappa_{1_{m+1}}\left(x_{1} b_{1}, \ldots, x_{1} b_{m+1}\right)=0,
$$

for all $b_{1}, \ldots, b_{m+1} \in \mathcal{B}$. The proof is complete.

3. Suppose that $A_{s}(n) \subseteq E(n) \subseteq A_{h}(n)$ for all $n$ and there exists a $k$ such that $E(k) \neq A_{s}(k)$. Let $\left\{u_{i, j}\right\}_{i, j=1, \ldots, k}$ be generators of $E(k)$. By proposition 3.5, $\exists i^{\prime}$ such that

$$
\sum_{l=1}^{k} u_{l, i^{\prime}}^{m} \neq 1
$$

for all odd numbers $m$. Without loss of generality, we assume that $i^{\prime}=1$. We need to show that $\kappa_{l}\left(x_{1} b_{1}, \ldots, x_{1} b_{l}\right)=0$ for all add numbers $l$ where $b_{1}, \ldots ., b_{l} \in B$. We prove this by induction on $l$. We have that

$$
\begin{aligned}
= & \sum_{\pi \in N C_{h}(l)} \sum_{\substack{\mathbf{i} \in[k]^{l} \\
\pi \leq \operatorname{ker} \mathbf{i}}}^{\mathbb{E}\left[x_{1} b_{1} \cdots x_{1} b_{l}\right] \otimes 1_{E(n)}} \kappa_{\pi}\left(x_{1} b_{1}, \ldots, x_{1} b_{l}\right) \otimes u_{\mathbf{i}, 1}+\sum_{\pi \in N C(l) \backslash N C_{h}(l)} \sum_{\substack{\mathbf{i} \in[k]^{l} \\
\pi \leq \operatorname{ker} \mathbf{i}}} \kappa_{\pi}\left(x_{1} b_{1}, \ldots, x_{1} b_{l}\right) \otimes u_{\mathbf{i}, 1} \\
= & \sum_{\pi \in N C_{h}(l)} \kappa_{\pi}\left(x_{1} b_{1}, \ldots, x_{1} b_{l}\right) \otimes 1_{E(n)}+\sum_{\pi \in N C(l) \backslash N C_{h}(l)} \sum_{\substack{\mathbf{i} \in[k]^{l} \\
\pi \leq \mathrm{ker} \mathbf{i}}} \kappa_{\pi}\left(x_{1} b_{1}, \ldots, x_{1} b_{l}\right) \otimes u_{\mathbf{i}, 1} .
\end{aligned}
$$

The first term of the last equality follows because $E(n)$ is a quotient algebra of $A_{h}(n)$. On the other hand, we have

$\mathbb{E}\left[x_{1} b_{1}, \ldots, x_{1} b_{l}\right] \otimes 1_{E(n)}=\sum_{\pi \in N C_{h}(l)} \kappa_{\pi}\left(x_{1} b_{1}, \ldots, x_{1} b_{l}\right) \otimes 1_{E(n)}+\sum_{\pi \in N C(l) \backslash N C_{h}(l)} \kappa_{\pi}\left(x_{1} b_{1}, \ldots, x_{1} b_{l}\right) \otimes 1_{E(n)}$.

Therefore,

$$
\sum_{\pi \in N C(l) \backslash N C_{h}(l)} \sum_{\substack{\mathbf{i} \in[k]^{l} \\ \pi \leq \operatorname{ker} \mathbf{i}}} \kappa_{\pi}\left(x_{1} b_{1}, \ldots, x_{1} b_{l}\right) \otimes u_{\mathbf{i}, 1}=\sum_{\pi \in N C(l) \backslash N C_{h}(l)} \kappa_{\pi}\left(x_{1} b_{1}, \ldots, x_{1} b_{l}\right) \otimes 1_{E(n)} .
$$

When $l=1$, we have $N C(1) \backslash N C_{h}(1)=\left\{1_{1}\right\}$, then

$$
\kappa^{(1)}\left(x_{1} b_{1}\right) \otimes\left(\sum_{l=1}^{k} u_{l, 1}-1_{E(n)}\right)=0 .
$$


Therefore, $\kappa_{1_{1}}\left(x_{1} b_{1}\right)=0$. Suppose $\kappa_{1_{l}}\left(x_{1} b_{1}, \ldots, x_{1} b_{l}\right)=0$ for odd numbers $l \leq 2 m$, then for $\pi \in N C(2 m+1), \kappa_{\pi}\left(x_{i_{1}} b_{1}, \ldots, x_{1} b_{2 m+1}\right)=0$ if $\pi$ contains a block whose size is an odd number less than $2 m$. Each partition $\pi \in N C(2 m+1)$ contains at least one block whose size is odd. Therefore, for $\pi \in N C(2 m+1), \kappa_{\pi}\left(x_{1} b_{1}, \ldots, x_{1} b_{2 m+1}\right)=0$ if $\pi \neq 1_{2 m+1}$. Hence, equation 2 becomes

$$
\kappa_{1_{2 m+1}}\left(x_{1} b_{1}, \ldots, x_{1} b_{2 m+1}\right) \otimes\left(\sum_{l=1}^{k} u_{l, 1}^{2 m+1}-1_{E(n)}\right)=0
$$

which implies

$$
\kappa_{1_{2 m+1}}\left(x_{1} b_{1}, \ldots, x_{1} b_{2 m+1}\right)=0,
$$

for all $b_{1}, \ldots, b_{m+1} \in \mathcal{B}$. The proof is complete.

4. If there exist $k_{1}, k_{2}$ such that $E\left(k_{1}\right) \nsubseteq A_{h}\left(k_{1}\right)$ and $E\left(k_{2}\right) \nsubseteq A_{b}\left(k_{2}\right)$, by Case 2 and Case 3 , the only non-vanishing cumulants are pair partition cumulants. The proof is done.

The proof of Theorem 1.1 follows the free case replacing noncrossing partitions by arbitrary partitions. The existence of the conditional expectation is a well known result in classical probability, see [9].

The proof of the Boolean case of Theorem 1.2 is slightly different. For Boolean de Finetti type theorem, we need to consider random variables in a $W^{*}$-probability space with a nondegenerated state $\phi[12$. In addition, we assume that $\mathcal{A}$ is generated by a sequence of random variables $\left(x_{i}\right)_{i \in \mathbb{N}}$. Let $\{E(n)\}_{n \in \mathbb{N}}$ be a sequence of orthogonal Boolean quantum groups such that $B_{s}(n) \subseteq E(n) \subseteq B_{o}(n)$ for each $n$. If the joint distribution of $\left(x_{i}\right)_{i \in \mathbb{N}}$ is $E(n)$-invariant, then the joint distribution of $\left(x_{i}\right)_{i \in \mathbb{N}}$ is $B_{s}(n)$ invariant for all $n$. By the main result in [12, there are a $W^{*}$-subalgebra $\mathcal{B}$ (not necessarily contain the unit of $\mathcal{A}$ ) such that $\mathcal{B} \subseteq \mathcal{A}$ and a $\phi$-preserving conditional expectation $\mathbb{E}: \mathcal{A} \rightarrow \mathcal{B}$ such that $\left(x_{i}\right)_{i \in \mathbb{N}}$ are Boolean independent and identically distributed with respect to $\mathbb{E}$. In this part of proof, we assume that $\mathcal{B}$ does not contain $1_{\mathcal{A}}$. Then, the tail algebra

$$
\mathcal{B}=\bigcap_{n=1}^{\infty} W^{*}\left\{x_{k} \mid k \geq n\right\}
$$

where $W^{*}\left\{x_{k} \mid k \geq n\right\}$ is the WOT closure of the non-unital algebra generated by $\left\{x_{k} \mid k \geq n\right\}$. We call $\mathcal{B}$ the non-unital tail algebra of $\left\{x_{i}\right\}_{i \in \mathbb{N}}$. Unlike the proof for free independence, the coaction invariant conditions for $\phi$ cannot be extended to the conditional expectation $E$ directly. Actually, we have a stronger statement.

Proposition 5.1. Let $(\mathcal{A}, \phi)$ be a $W^{*}$-probability space and $\left(x_{i}\right)_{i \in \mathbb{N}}$ be a sequence of selfadjoint random variables which generate $\mathcal{A}$ as a von Neumann algebra and the unit of $\mathcal{A}$ is contained in the WOT closure of the non-unital algebra generated by $\left(x_{i}\right)_{i \in \mathbb{N}}$. Let $E(n)$ be a sequence of Boolean orthogonal quantum semigroups such that $B_{s}(n) \subseteq E(n) \subseteq B_{o}(n)$. If $\left(x_{i}\right)_{i \in \mathbb{N}}$ are $E(n)$-invariant for all $n$, then there exists a $\phi$-preserving conditional expectation $\mathbb{E}: \mathcal{A} \rightarrow \mathcal{B}$, where $\mathcal{B}$ is the non-unital tail algebra of $\left\{x_{i}\right\}_{i \in \mathbb{N}}$, such that $\left(x_{i}\right)_{i \in \mathbb{N}}$ is Boolean independent with respect to $\mathbb{E}$. Let $\mathcal{A}_{n}$ be the non-unital algebra generated by $\left\{x_{i}\right\}_{i \in \mathbb{N}}$. We have that

$$
\mathbb{E}\left[a_{1} b a_{2}\right]=\mathbb{E}\left[a_{1}\right] b \mathbb{E}\left[a_{2}\right],
$$

where $a_{1}, a_{2} \in \mathcal{A}_{n}$ for some $n$ and $b \in \mathcal{B}$. Let $\left\{u_{i, j}\right\}_{i, j=1, \ldots, n}$ and $\mathbf{P}$ be the generators of $E(n)$. We have that

$$
\mathbb{E}\left[x_{i_{1}} \cdots x_{i_{k}}\right] \otimes \mathbf{P}=\sum_{j_{1}, \ldots, j_{k}=1}^{n} \mathbb{E}\left[x_{j_{1}} \cdots x_{j_{k}}\right] \otimes u_{j_{1}, i_{1}} \cdots u_{j_{k}, i_{k}} \mathbf{P}
$$

for $i_{1}, \ldots, i_{k} \leq n$. 
Proof. The existence of $\mathbb{E}$ is shown in [12]. We turn to prove the two equations of this proposition. Given $a_{1}, a_{2} \in \mathcal{A}_{n}$ for some $n$ and $b \in \mathcal{B}$, by assumption, $b$ is contained in the $W^{*}$-closure of the non-unital algebra generated by $\left\{x_{i} \mid i>n\right\}$. By Kaplansky theorem, there exist a net of bounded elements $\left(y_{i}\right)_{i \in \omega}$ such that $\left(y_{i}\right)_{i \in \omega}$ are contained in the non-unital algebra generated by $\left\{x_{i} \mid i>n\right\}$ and converge to $b$ in strong operator topology. Therefore, by normality of $\mathbb{E}$, we have

$$
\mathbb{E}\left[a_{1} b a_{2}\right]=\lim _{i \rightarrow \infty} \mathbb{E}\left[a_{1} y_{i} a_{2}\right]=\lim _{i \rightarrow \infty} \mathbb{E}\left[a_{1}\right] \mathbb{E}\left[y_{i}\right] \mathbb{E}\left[a_{2}\right]=\mathbb{E}\left[a_{1}\right] b \mathbb{E}\left[a_{2}\right],
$$

where the second equality follows because $\left(x_{i}\right)_{i \in \mathbb{N}}$ are Boolean independent with respect to $\mathbb{E}$. The second equation of this proposition can be checked pointwisely. Let $a_{1}, a_{2} \in \mathcal{A}_{m}$ for some $m$. In [12], it was shown that there exists a normal homomorphism $\alpha: \mathcal{A} \rightarrow \mathcal{A}$ such that $\alpha\left(x_{i}\right)=x_{i+1}$ for all $i \in \mathbb{N}$. By the proof of Lemma 6.7 in [12] and the assumption that $\left\{x_{i}\right\}_{i \in \mathbb{N}}$ is $E(n)$-invariant, we have

$$
\begin{aligned}
& \phi\left(a_{1} \mathbb{E}\left[x_{i_{1}} \cdots x_{i_{k}}\right] a_{2}\right) \otimes \mathbf{P} \\
= & \lim _{l \rightarrow \infty, l>m} \phi\left(a_{1} \alpha^{l}\left(x_{i_{1}} \cdots x_{i_{k}}\right) a_{2}\right) \otimes \mathbf{P} \\
= & \lim _{l \rightarrow \infty, l>m} \phi\left(\alpha^{n}\left(a_{1}\right) x_{i_{1}} \cdots x_{i_{k}} \alpha^{n}\left(a_{2}\right)\right) \otimes \mathbf{P} \\
= & \lim _{l \rightarrow \infty, l>m}\left(\phi\left(\alpha^{n}\left(a_{1}\right) \sum_{j_{1}, \ldots, j_{k}=1}^{n} x_{j_{1}} \cdots x_{j_{k}} \alpha^{n}\left(a_{2}\right)\right) \otimes u_{j_{1}, i_{1}} \cdots u_{j_{k}, i_{k}} \mathbf{P}\right. \\
= & \lim _{l \rightarrow \infty, l>m} \phi\left(a_{1} \alpha^{l}\left(\sum_{j_{1}, \ldots, j_{k}=1}^{n} x_{j_{1}} \cdots x_{j_{k}}\right) a_{2}\right) \otimes u_{j_{1}, i_{1}} \cdots u_{j_{k}, i_{k}} \mathbf{P} \\
= & \sum_{j_{1}, \ldots, j_{k}=1}^{n} \phi\left(a_{1} \mathbb{E}\left[x_{j_{1}} \cdots x_{j_{k}}\right] a_{2}\right) \otimes u_{j_{1}, i_{1}} \cdots u_{j_{k}, i_{k}} \mathbf{P} .
\end{aligned}
$$

Since $a_{1}, a_{2}$ are arbitrarily chosen from the dense set $\bigcup_{n \rightarrow \infty} \mathcal{A}_{n}$ of $\mathcal{A}$, the proof is done.

Now, we are ready to prove Boolean case of Theorem 1.2

Proof. Statement 1. This is the Boolean de Finetti theorem in [12].

Statement 2. As the free case, we need to show that $b_{\mathbb{E}}^{(l)}\left(x_{1} b_{1}, \ldots ., x_{1} b_{l}\right)=0$ for all $l \geq 3$ where $b_{1}, \ldots, b_{l} \in B \cup\left\{\mathbb{C} 1_{\mathcal{A}}\right\}$. By proposition 5.1, we have

$$
\begin{aligned}
& \mathbb{E}\left[x_{\mathbf{i}_{1}} b_{1} x_{\mathbf{i}_{2}} \cdots b_{n-1} x_{\mathbf{i}_{m}}\right] \\
= & \mathbb{E}\left[x_{\mathbf{i}_{1}}\right] b_{1} \mathbb{E}\left[x_{\mathbf{i}_{2}}\right] \cdots b_{n-1} \mathbb{E}\left[x_{\mathbf{i}_{m}}\right] \\
= & \sum_{\pi_{1} \in I\left(k_{1}\right)} b_{\mathbb{E}}^{\left(\pi_{1}\right)}\left(x_{i_{1}^{(1)}}, \ldots x_{\left.i_{k_{1}}^{(1)}\right)} b_{1} \sum_{\pi_{2} \in I\left(k_{2}\right)} b_{\mathbb{E}}^{\left(\pi_{2}\right)}\left(x_{i_{1}^{(2)}}, \ldots x_{i_{k_{2}}(2)}\right) \cdots b_{n-1} \sum_{\pi_{m} \in I\left(k_{m}\right)} b_{\mathbb{E}}^{\left(\pi_{m}\right)}\left(x_{\left.i_{1}^{(m)}, \ldots x_{i_{k_{m}}}\right)}\right)\right. \\
= & \sum_{\pi \in I\left(k_{1}\right) \times I\left(k_{2}\right) \times \cdots \times I\left(k_{m}\right)} b_{\mathbb{E}}^{(\pi)}\left(x_{i_{1}^{(1)}}, \ldots x_{i_{k_{1}}^{(1)}}, b_{1} x_{i_{1}^{(2)}}, \ldots x_{i_{k_{2}}^{(2)}}, \cdots, b_{n-1} x_{i_{1}^{(m)}}, \ldots x_{i_{k_{m}}(m)}\right),
\end{aligned}
$$

where $\mathbf{i}_{l}=\left(i_{1}^{(l)}, \ldots, i_{k_{l}}^{(l)}\right) \in[n]^{k_{l}}$ for all $l=1, \ldots, m$ for some $n$ and $b_{1}, \ldots, b_{m} \in \mathcal{B}$. Therefore, to finish the proof, we need to show that $b_{\mathbb{E}}^{(k)}\left(x_{1}, \ldots, x_{1}\right)=0$ for all $l \geq 3$. The rest of the poof is similar to the free case: 
Let $\left\{u_{i, j}\right\}_{i, j=1, \ldots, k}$ 's and $\mathbf{P}$ be the generators of $E(k)$. First, by Proposition [5.1, we have

$$
\begin{aligned}
& \mathbb{E}\left[x_{1} \cdots x_{1}\right] \otimes \mathbf{P} \\
= & \sum_{\mathbf{i} \in[k]^{l}} \mathbb{E}\left[x_{\mathbf{i}}\right] \otimes u_{\mathbf{i}, 1} \mathbf{P} \\
= & \sum_{\mathbf{i} \in[k]^{l}} \sum_{\pi \in I(l)} b_{\mathbb{E}}^{(\pi)}\left(x_{\mathbf{i}}\right) \otimes u_{\mathbf{i}, 1} \\
= & \sum_{\pi \in I_{b}(l)} \sum_{\mathbf{i} \in[k]^{l}} b_{\mathbb{E}}^{(\pi)}\left(x_{i_{1}}, \ldots, x_{i_{l}}\right) \otimes u_{\mathbf{i}, 1} \mathbf{P}+\sum_{\pi \in I(l) \backslash I_{b}(l)} \sum_{\mathbf{i} \in[k]^{l}} b_{\mathbb{E}}^{(\pi)}\left(x_{i_{1}}, \ldots, x_{i_{l}}\right) \otimes u_{\mathbf{i}, 1} \mathbf{P} \\
= & \sum_{\pi \in I_{b}(l)} \sum_{\substack{\mathbf{i} \in[k]^{l} \\
\pi \leq \operatorname{ker} \mathbf{i}}} b_{\mathbb{E}}^{(\pi)}\left(x_{i_{1}}, \ldots, x_{i_{l}}\right) \otimes u_{\mathbf{i}, 1} \mathbf{P}+\sum_{\pi \in I(l) \backslash I_{b}(l)} \sum_{\substack{\mathbf{i} \in[k]^{l} \\
\pi \leq k e r}} b_{\mathbb{E}}^{(\pi)}\left(x_{i_{1}}, \ldots, x_{i_{l}}\right) \otimes u_{\mathbf{i}, 1} \mathbf{P} \\
= & \sum_{\pi \in I_{b}(l)} \sum_{\substack{\mathbf{i} \in[k]^{l} \\
\pi \leq \operatorname{ker} \mathbf{i}}} b_{\mathbb{E}}^{(\pi)}\left(x_{1}, \ldots, x_{1}\right) \otimes u_{\mathbf{i}, 1} \mathbf{P}+\sum_{\pi \in I(l) \backslash I_{b}(l)} \sum_{\substack{\mathbf{i} \in[k]^{l} \\
\pi \leq \operatorname{ker} \mathbf{i}}} b_{\mathbb{E}}^{(\pi)}\left(x_{1}, \ldots, x_{1}\right) \otimes u_{\mathbf{i}, 1} \mathbf{P} \\
= & \sum_{\pi \in I_{b}(l)} b_{\mathbb{E}}^{(\pi)}\left(x_{1} b_{1}, \ldots, x_{1} b_{l}\right) \otimes \mathbf{P}+\sum_{\pi \in I(l) \backslash I_{b}(l)} \sum_{\substack{\mathbf{i} \in[k]^{l} \\
\pi \leq \operatorname{ker} \mathbf{i}}} b_{\mathbb{E}}^{(\pi)}\left(x_{1}, \ldots, x_{1}\right) \otimes u_{\mathbf{i}, 1} \mathbf{P} .
\end{aligned}
$$

The first term of the last equality follows because $E(n)$ is a quotient algebra of $B_{b}(n)$. On the other hand

$$
\mathbb{E}\left[x_{1}, \ldots, x_{1}\right] \otimes \mathbf{P}=\sum_{\pi \in I_{b}(k)} b_{\mathbb{E}}^{(\pi)}\left(x_{1}, \ldots, x_{1}\right) \otimes \mathbf{P}+\sum_{\pi \in I(l) \backslash I_{b}(l)} b_{\mathbb{E}}^{(\pi)}\left(x_{1}, \ldots, x_{1}\right) \otimes \mathbf{P} .
$$

Therefore,

$$
\sum_{\pi \in I(l) \backslash I_{b}(l)} \sum_{\substack{\mathbf{i} \in[k]^{l} \\ \pi \leq \mathrm{ker} \mathbf{i}}} b_{\mathbb{E}}^{(\pi)}\left(x_{1}, \ldots, x_{1}\right) \otimes u_{\mathbf{i}, 1} \mathbf{P}=\sum_{\pi \in I(l) \backslash I_{b}(l)} b_{\mathbb{E}}^{(\pi)}\left(x_{1}, \ldots, x_{1}\right) \otimes \mathbf{P} .
$$

By assumption, $E(k)$ has a quotient algebra $E^{\prime}(k)$ such that $A_{s}(k) \nsubseteq E^{\prime}(k) \subseteq A_{n}(n)$. Let $\left\{u_{i, j}^{\prime}\right\}$ be the generators of $E^{\prime}(k)$. Then, there exists a $C^{*}$-homomorphism $\Psi: E(k) \rightarrow E^{\prime}(k)$ such that

$$
\Psi\left(u_{i, j}\right)=u_{i, j}^{\prime} \text { for all } i, j=1, \ldots, k, \text { and } \Psi(\mathbf{P})=1_{E^{\prime}(k)} .
$$

Without loss of generality, by proposition 3.5, we can assume that

$$
\sum_{l=1}^{k}\left(u_{l, 1}^{\prime}\right)^{m} \neq 1
$$

for all $m>2$. Let $i d \otimes \Psi$ acts on equation 4 . Then, we get

$$
\sum_{\pi \in I(l) \backslash I_{b}(l)} \sum_{\substack{\mathbf{i} \in[k]^{l} \\ \pi \leq \operatorname{ker} \mathbf{i}}} b_{\mathbb{E}}^{(\pi)}\left(x_{1}, \ldots, x_{1}\right) \otimes u_{\mathbf{i}, 1}^{\prime}=\sum_{\pi \in I(l) \backslash I_{b}(l)} b_{\mathbb{E}}^{(\pi)}\left(x_{1}, \ldots, x_{1}\right) \otimes 1_{E^{\prime}(k)} .
$$

When $l=3$, we have $I(3) \backslash I_{b}(3)=\left\{1_{3}\right\}$, then

$$
\sum_{\substack{\mathbf{i} \in[n]^{k} \\ \pi \leq \operatorname{ker} 1_{3}}} b_{\mathbb{E}}^{(3)}\left(x_{1}, \ldots, x_{1}\right) \otimes u_{\mathbf{i}, 1}^{\prime}=b_{\mathbb{E}}^{(3)}\left(x_{1}, \ldots, x_{1}\right) \otimes 1_{E^{\prime}(k)},
$$


which is

$$
\kappa_{1_{3}}\left(x_{1}, \ldots, x_{1}\right) \otimes\left(\sum_{l=1}^{k} u_{l, 1}^{\prime 3}-1_{E^{\prime}(k)}\right)=0 .
$$

Therefore, $b_{\mathbb{E}}^{(3)}\left(x_{1}, \ldots, x_{1}\right)=0$. Suppose $b_{\mathbb{E}}^{(l)}\left(x_{1} b_{1}, \ldots, x_{1} b_{l}\right)=0$ for $3 \leq l \leq m$. Then, for $\pi \in$ $I(m+1), b_{E}^{(\pi)}\left(x_{1}, \ldots, x_{1}\right)=0$ if $\pi$ contains a block whose size is between 3 and $m$. Each partition $\pi \in I(m+1) \backslash I_{b}(m+1)$ contains at least one block whose size is greater than 2 . Therefore, for $\pi \in I(m+1) \backslash I_{b}(m+1), b_{E}^{(\pi)}\left(x_{1}, \ldots, x_{1}\right)=0$ if $\pi \neq 1_{m+1}$. Hence, equation 1 becomes

$$
b_{E}^{(m+1)}\left(x_{1}, \ldots, x_{1}\right) \otimes\left(\sum_{l=1}^{k} u_{l, 1}^{\prime m+1}-1_{E^{\prime}(k)}\right)=0
$$

which implies

$$
b_{E}^{(m+1)}\left(x_{1}, \ldots, x_{1}\right)=0 .
$$

The proof of Statement 2 is complete.

Similarly, by the proof of Statement 3 and Statement 4 for free case, Statement 3 and Statement 4 for Boolean independence are also true.

Remark 5.2. Our general de Finetti theorem for Boolean independence is not complete since we know very little about the classification of Boolean quantum semigroups.

According the diagrams in Section 3 and Section 4, we have the following relations.

1. $C_{s}(n) \subseteq C_{s^{\prime}}(n) \subseteq C_{b}(n)$ for all $\mathrm{n}$, and $C_{s}(n) \neq C_{s^{\prime}}(n)$ for $n>3$.

2. $C_{b^{\prime}}(n) \nsubseteq C_{h}(n)$ and $C_{b^{\prime}}(n) \nsubseteq C_{b}(n)$ for $n>3$.

3. $A_{s} n \subseteq A_{s^{\prime}}(n) \subseteq A_{b}(n)$ for all $\mathrm{n}$, and $A_{s}(n) \neq A_{s^{\prime}}(n)$ for $n>3$.

4. $A_{b^{\prime}}(n), A_{b^{\#}}(n) \nsubseteq A_{h}(n)$ and $A_{b^{\prime}}(n), A_{b^{\#}}(n) \nsubseteq A_{b}(n)$ for $n>3$.

5. $B_{s}(n) \subseteq B_{s^{\prime}}(n) \subseteq B_{b}(n)$ for all $\mathrm{n}$, and $B_{s}(n) \neq B_{s^{\prime}}(n)$ for $n>3$. Moreover $A_{s^{\prime}}(n)$ is a quotient algebra of $B_{s^{\prime}}(n)$

6. $A_{b^{\prime}}$ is a quotient algebra of $B_{b^{\prime}}(n)$ for $n>3$.

By Theorem 1.1, we have the following corollary for classical independence.

Corollary 5.3. Let $(\Omega, \Sigma, \mu)$ be a classical probability space and $\left(x_{i}\right)_{i \in \mathbb{N}}$ be a sequence of realvalued random variables such that $x_{i} \in \bigcap_{1 \leq p<\infty} L^{p}(\Omega, d \mu)$ for all $i$. Let $\mathcal{A}$ be the algebra of all random variables and $\phi$ is the expectation. Assume that $\left(x_{i}\right)_{i \in \mathbb{N}}$ generate $\mathcal{A}$.

1. If the joint distribution of $\left(x_{i}\right)_{i \in \mathbb{N}}$ is $C_{s^{\prime}}(n)$ invariant for all $n \in \mathbb{N}$, then there exist a subalgebra $\mathcal{B}$ and a $\phi$-preserving conditional expectation $\mathbb{E}: \mathcal{A} \rightarrow \mathcal{B}$ such that such that $\left(x_{i}\right)_{i \in \mathbb{N}}$ are conditionally independent and have identically symmetric distribution with respect to $\mathbb{E}$.

2. If the joint distribution of $\left(x_{i}\right)_{i \in \mathbb{N}}$ is $C_{b^{\prime}}(n)$ invariant for all $n \in \mathbb{N}$, then there exist a subalgebra $\mathcal{B}$ and a $\phi$-preserving conditional expectation $\mathbb{E}: \mathcal{A} \rightarrow \mathcal{B}$ such that such that $\left(x_{i}\right)_{i \in \mathbb{N}}$ are conditionally independent and have centered Gaussian distribution with respect to $\mathbb{E}$.

By Theorem 1.2, we have the following result for free and Boolean independence.

Corollary 5.4. Let $(\mathcal{A}, \phi)$ be a $W^{*}$-probability space and $\left(x_{i}\right)_{i \in \mathbb{N}}$ be a sequence of random variables which generate $\mathcal{A}$.

- Free case: Suppose that $\phi$ is faithful. 
1. If the joint distribution of $\left(x_{i}\right)_{i \in \mathbb{N}}$ is $A_{s^{\prime}}(n)$ invariant for all $n \in \mathbb{N}$, then there exist a $W^{*}$-subalgebra $1 \subseteq \mathcal{B} \subseteq \mathcal{A}$ and a $\phi$-preserving conditional expectation $\mathbb{E}: \mathcal{A} \rightarrow \mathcal{B}$ such that $\left(x_{i}\right)_{i \in \mathbb{N}}$ are freely independent and have identically symmetric distribution with respect to $\mathbb{E}$.

2. If the joint distribution of $\left(x_{i}\right)_{i \in \mathbb{N}}$ is $A_{b^{\prime}}(n)$-invariant for all $n \in \mathbb{N}$, then exist a $W^{*}$-subalgebra $1 \subseteq \mathcal{B} \subseteq \mathcal{A}$ and a $\phi$-preserving conditional expectation $\mathbb{E}: \mathcal{A} \rightarrow \mathcal{B}$ such that $\left(x_{i}\right)_{i \in \mathbb{N}}$ are freely independent and have centered semicircular distribution with respect to $\mathbb{E}$.

3. If the joint distribution of $\left(x_{i}\right)_{i \in \mathbb{N}}$ is $A_{b^{\#}}(n)$-invariant for all $n \in \mathbb{N}$, then exist a $W^{*}$-subalgebra $1 \subseteq \mathcal{B} \subseteq \mathcal{A}$ and a $\phi$-preserving conditional expectation $\mathbb{E}: \mathcal{A} \rightarrow \mathcal{B}$ such that $\left(x_{i}\right)_{i \in \mathbb{N}}$ are freely independent and have centered semicircular distribution with respect to $\mathbb{E}$.

- Boolean case: If $\phi$ is non-degenerated.

1. If the joint distribution of $\left(x_{i}\right)_{i \in \mathbb{N}}$ is $B_{s^{\prime}}(n)$ invariant for all $n \in \mathbb{N}$, then there are a $W^{*}$-subalgebra(not necessarily contain the unit of $\left.\mathcal{A}\right) \mathcal{B} \subseteq \mathcal{A}$ and a $\phi$-preserving conditional expectation $\mathbb{E}: \mathcal{A} \rightarrow \mathcal{B}$ such that $\left(x_{i}\right)_{i \in \mathbb{N}}$ are Boolean independent and have identically symmetric distribution with respect to $\mathbb{E}$.

2. If the joint distribution of $\left(x_{i}\right)_{i \in \mathbb{N}}$ is $B_{b^{\prime}}(n)$ invariant for all $n \in \mathbb{N}$, then there are a $W^{*}$-subalgebra(not necessarily contain the unit of $\left.\mathcal{A}\right) \mathcal{B} \subseteq \mathcal{A}$ and a $\phi$-preserving conditional expectation $\mathbb{E}: \mathcal{A} \rightarrow \mathcal{B}$ such that $\left(x_{i}\right)_{i \in \mathbb{N}}$ are conditionally independent and have centered Bernoulli distribution with respect to $\mathbb{E}$.

Acknowledgment The author would like to thank his thesis advisor, D.-V. Voiculescu, for his continued guidance and support while completing this project. While working on this paper, the author was supported in part by funds from NSF grant DMS-1301727.

\section{REFERENCES}

[1] Octavio Arizmendi, Takahiro Hasebe, Franz Lehner, and Carlos Vargas. Relations between cumulants in noncommutative probability. Adv. Math., 282:56-92, 2015.

[2] Teodor Banica, Stephen Curran, and Roland Speicher. Classification results for easy quantum groups. Pacific J. Math., 247(1):1-26, 2010.

[3] Teodor Banica, Stephen Curran, and Roland Speicher. De Finetti theorems for easy quantum groups. Ann. Probab., 40(1):401-435, 2012.

[4] Marek Bożejko and Roland Speicher. $\psi$-independent and symmetrized white noises. In Quantum probability $\& 6$ related topics, QP-PQ, VI, pages 219-236. World Sci. Publ., River Edge, NJ, 1991.

[5] Stephen Curran. Quantum rotatability. Trans. Amer. Math. Soc., 362(9):4831-4851, 2010.

[6] David A. Freedman. Invariants under mixing which generalize de Finetti's theorem: Continuous time parameter. Ann. Math. Statist., 34:1194-1216, 1963.

[7] Tomohiro Hayase. De Finetti theorems for a Boolean analogue of easy quantum groups. J. Math. Sci. Univ. Tokyo, 24(3):355-398, 2017.

[8] Olav Kallenberg. Spreading-invariant sequences and processes on bounded index sets. Probab. Theory Related Fields, 118(2):211-250, 2000.

[9] Olav Kallenberg. Probabilistic symmetries and invariance principles. Probability and its Applications (New York). Springer, New York, 2005.

[10] Claus Köstler and Roland Speicher. A noncommutative de Finetti theorem: invariance under quantum permutations is equivalent to freeness with amalgamation. Comm. Math. Phys., 291(2):473-490, 2009.

[11] Franz Lehner. Cumulants in noncommutative probability theory. I. Noncommutative exchangeability systems. Math. Z., 248(1):67-100, 2004.

[12] Weihua Liu. A noncommutative de Finetti theorem for boolean independence. J. Funct. Anal., 269(7):19501994, 2015.

[13] Alexandru Nica and Roland Speicher. Lectures on the combinatorics of free probability, volume 335 of London Mathematical Society Lecture Note Series. Cambridge University Press, Cambridge, 2006. 
[14] Mihai Popa. A new proof for the multiplicative property of the Boolean cumulants with applications to the operator-valued case. Colloq. Math., 117(1):81-93, 2009.

[15] C. Ryll-Nardzewski. On stationary sequences of random variables and the de Finetti's equivalence. Colloq. Math., 4:149-156, 1957.

[16] Piotr M. Soltan. Quantum families of maps and quantum semigroups on finite quantum spaces. J. Geom. Phys., 59(3):354-368, 2009.

[17] Piotr Mikołaj Sołtan. On quantum semigroup actions on finite quantum spaces. Infin. Dimens. Anal. Quantum Probab. Relat. Top., 12(3):503-509, 2009.

[18] Roland Speicher. On universal products. In Free probability theory (Waterloo, ON, 1995), volume 12 of Fields Inst. Commun., pages 257-266. Amer. Math. Soc., Providence, RI, 1997.

[19] Roland Speicher. Combinatorial theory of the free product with amalgamation and operator-valued free probability theory. Mem. Amer. Math. Soc., 132(627):x+88, 1998.

[20] Roland Speicher and Reza Woroudi. Boolean convolution. In Free probability theory (Waterloo, ON, 1995), volume 12 of Fields Inst. Commun., pages 267-279. Amer. Math. Soc., Providence, RI, 1997.

[21] Şerban Strătilă. Modular theory in operator algebras. Editura Academiei Republicii Socialiste România, Bucharest; Abacus Press, Tunbridge Wells, 1981. Translated from the Romanian by the author.

[22] D. V. Voiculescu, K. J. Dykema, and A. Nica. Free random variables, volume 1 of CRM Monograph Series. American Mathematical Society, Providence, RI, 1992. A noncommutative probability approach to free products with applications to random matrices, operator algebras and harmonic analysis on free groups.

[23] Shuzhou Wang. Free products of compact quantum groups. Comm. Math. Phys., 167(3):671-692, 1995.

[24] Shuzhou Wang. Quantum symmetry groups of finite spaces. Comm. Math. Phys., 195(1):195-211, 1998.

[25] Moritz Weber. On the classification of easy quantum groups. Adv. Math., 245:500-533, 2013.

[26] S. L. Woronowicz. Compact matrix pseudogroups. Comm. Math. Phys., 111(4):613-665, 1987.

[27] S. L. Woronowicz. Unbounded elements affiliated with $C^{*}$-algebras and noncompact quantum groups. Comm. Math. Phys., 136(2):399-432, 1991.

Department of Mathematics

Indiana University

Bloomington, IN 47401, USA

E-MAIL: liuweih@indiana.edu 